\title{
A fast frequency sweep approach using Padé approximations for solving Helmholtz finite element models
}

\author{
Marcos Souza Lenzi ${ }^{\mathrm{a}, \mathrm{b}}$, Sanda Lefteriu ${ }^{\mathrm{a}}$, Hadrien Beriot ${ }^{\mathrm{a}, *}$, Wim Desmet $^{\mathrm{b}}$ \\ a LMS International, Interleuvenlaan 68, 3001 Leuven, Belgium \\ ${ }^{\mathrm{b}} \mathrm{KU}$ Leuven, Department of Mechanical Engineering, Division PMA, Celestijnenlaan 300b - bus 2420, 3001 Leuven, Belgium
}

\section{A R T I C L E I N F O}

\section{Article history:}

Accepted 29 May 2012

Handling Editor: J. Astley

\begin{abstract}
A B S T R A C T
In various engineering applications, the solution of the Helmholtz equation is required over a broad frequency range. The simplest approach, which consists in solving the system of equations obtained from a finite element discretization for each frequency, becomes computationally prohibitive for fine increments, particularly when dealing with large systems, like those encountered when addressing mid-frequency problems. Alternative approaches involving reduced-order models built via Padé approximations are now well established for systems exhibiting polynomial frequency dependency of second-order kind and for frequency independent excitations. This paper treats systems of more complicated wavenumber dependency, likely to be encountered when applying frequency dependent boundary conditions and/or loadings. The well-conditioned asymptotic waveform evaluation (WCAWE) is selected as the method of choice and the approximated Taylor coefficients are computed by differentiating the continuous frequency dependent models obtained through a fitting process of the system entries. The method is benchmarked first against the Second-Order Arnoldi (SOAR) algorithm on a simple second-order system. Then it is applied to realistic large scale interior and exterior Helmholtz problems exhibiting high-order polynomial or rational frequency behavior. In either case, the proposed methodology is shown to reduce the computational time of the frequency sweep by an order of magnitude when compared to the direct approach.
\end{abstract}

(c) 2012 Elsevier Ltd. All rights reserved.

\section{Introduction}

Frequency sweeps over a wide frequency range and for a large number of frequencies are required in many applications in acoustics, like car cavity design as interior problems or radiation of vibrating structures as exterior problems. Since the complex symmetric matrix arising from the finite element discretization of the Helmholtz problem is wavenumber dependent, computing the solution generally involves a separate inversion at each frequency, leading to a computational cost which grows linearly with the number of frequency evaluations. When dealing with large systems, like those arising when tackling mid-frequency applications, this becomes computationally expensive, if not prohibitive.

\footnotetext{
* Corresponding author. Tel.: +32 16384 403; fax: +32 16384505 .

E-mail addresses: marcos.souzalenzi@Imsintl.com (M. Souza Lenzi), sanda.lefteriu@lmsintl.com (S. Lefteriu), hadrien.beriot@lmsintl.com (H. Beriot), Wim.Desmet@mech.kuleuven.be (W. Desmet).
} 
Modal superposition has been the preferred method in commercial software in the past decades to reduce the computational burden associated to the original problem. The eigenmodes of interest are computed once, leading to a reduced-order model obtained through an appropriate projection, which can be easily inverted at a large number of frequencies [1]. Furthermore, the eigenmodes provide useful physical insights to engineers on the global behavior of the dynamical system. However, the modal superposition technique is only efficient when applied to systems with polynomial frequency dependency of first-order kind (standard state-space or descriptor systems) which renders it inappropriate for many applications. Moreover, in the mid-frequency range, the modal density and modal overlap increase, causing the modal superposition to become less effective.

As an alternative to modal superposition, fast frequency sweep (FFS) methods have received a considerable attention in the engineering community. The objective of FFS is to accelerate the computation of the frequency response such that the solution can be obtained at a much larger number of points for the same computational effort. The basic idea is to compute the solution at a few key frequencies (expansion points) and then determine the behavior at intermediary points by extrapolating the response in the neighborhood. The extrapolation is based on the knowledge of the derivatives of the solution evaluated at the expansion points (also called moments).

One moment matching approach which has received particular attention is the Padé type approximation [2]. This technique approximates the response around an expansion point by a rational function of a given order by matching terms in the Taylor series expansion. The use of Padé approximation versus more classical Taylor series expansions is motivated by the fact that the response function has singular points (poles), where the Taylor series does not converge.

Padé approximants can be computed by matching the moments in an implicit way via Krylov subspace projections. Padé-via-Lanczos (PVL) [3,4] and matrix Padé-via-Lanczos [5,6] achieve moment matching by building Krylov subspaces using the Lanczos process. Krylov methods were shown to lead to substantial improvements for large scale multifrequency analysis in various engineering applications [7-11].

Moment matching for systems with first-order polynomial frequency dependency in the system matrix is well understood. However, its application to systems of second-order kind with mass, stiffness and constant non-proportional damping systems is only possible through a linearization, which doubles the dimension of the original problem and increases memory requirements substantially [12]. Therefore, researchers developed methods to solve the quadratic eigenvalue problem directly [13-15]. A major breakthrough was achieved in 2005 by the so-called Second-order Arnoldi (SOAR) algorithm [16], as it allows for an elegant and efficient way of reducing second-order systems while preserving their second-order structure. It is based on a generalization of the standard Krylov subspace to a second-order Krylov subspace, constructed from one initial vector and two particular matrices. Several improvements and extensions of SOAR can be found in the literature, like the "Refined Second-Order Arnoldi (RSOAR)" [17], "SAPOR: Second-Order Arnoldi method for Passive Order Reduction" [18] and the "Two-Sided Second-Order Arnoldi Algorithm" [19-21].

In this paper, the focus is on problems with more complicated frequency behavior of the system matrix and of the righthand side. In many real-life engineering applications involving Helmholtz solutions, some properties of the model (impedance, loadings,...) are provided under the form of a set of data points and their frequency behavior is not known explicitly. Furthermore, for exterior Helmholtz applications, modeling the unbounded domain requires the introduction of a specific operator on the truncation surface which is typically frequency dependent. Although Padé-via-Lanczos may be implemented in combination with a Dirichlet-to-Neumann (DtN) non-reflecting map [22], this can only be done through an ad hoc modification of the algorithm. In the general case, the frequency dependency may be too complicated for adapting Krylov methods. Here, radiation problems are tackled through the introduction of locally conformal Perfectly Matched Layers (PMLs) around the computational domain [23], which are shown to yield a matrix equation with rational frequency dependency.

For such non-second-order systems, methods based on direct Padé approximations have to be employed. The first method applying Padé approximation was the Asymptotic Waveform Evaluation (AWE) [24], which computes and matches moments explicitly. The approximants are computed by setting up a Hankel linear system, whose solution determines the coefficients of the rational function [25]. This approach was successful in reducing the complexity of systems arising from a finite element method (FEM) discretization of electromagnetic systems [26]. However, this Hankel system is inherently ill-conditioned, which limits the expansion to moderate orders [2]. As an alternative, Galerkin AWE (GAWE) [31] enforces a Galerkin treatment and, unlike AWE, GAWE does not form the Padé approximants explicitly. Instead, the original matrix is projected onto the subspace formed by the orthonormalized moments and a Galerkin condition is imposed, making the residual of the approximation orthogonal to the moments subspace.

Nevertheless, both AWE and GAWE compute the moments explicitly through an ill-conditioned vector-generation process and therefore exhibit a small bandwidth of convergence. To alleviate this particular issue, Slone [28] introduced the wellconditioned AWE (WCAWE) algorithm. This implicit moment matching process generates moments that do not stagnate, thanks to the introduction of correction factors, yielding an approximation with a significantly wider bandwidth [29].

Padé approximation is intrinsically a single frequency expansion technique. To apply it over a wide frequency range, a multi-expansion point strategy must be implemented to render an efficient FFS algorithm [30]. For instance, a multi-point extension of GAWE (MGAWE) was introduced by Slone et al. [31], while the Adaptive Windowing Algorithm (AWA), developed by Tuck-Lee et al. [10] and applied in conjunction with the block Lanczos algorithm, is another example. In Lenzi et al. [32], the combination of AWA and SOAR was able to provide reduced-order models with good levels of accuracy over a wide frequency range. 
The objective of the paper is to propose a more general approach to accelerate the computation of Helmholtz interior or exterior FFS problems, including those involving non-explicit frequency dependencies in the matrix and right-hand side entries. As no differentiation can be performed in symbolic closed form, a system identification procedure which constructs function approximations for all system entries is proposed to approximate the Taylor coefficients of the solution. The subspace computed from this system identification step is then used in the WCAWE algorithm coupled with an AWA multi-point strategy. Though the examples and formulations presented here assume only uncoupled acoustics problems in quiescent medium, it is believed that it could be also applied for coupled vibro-acoustic or convected acoustics applications.

The paper is structured as follows. Section 2 presents the interior and exterior Helmholtz boundary value problems and discusses the frequency dependency of the resulting algebraic systems. Section 3 introduces the WCAWE algorithm, while Section 4 discusses its application to systems with complicated frequency dependency. In Section 5, the WCAWE algorithm is coupled with a multiple expansion points strategy. Finally, in Section 6, the proposed approach is validated on several academic and industrial test cases, while Section 7 concludes the paper.

\section{Problem description}

This section introduces the interior and exterior Helmholtz boundary value problems and their finite element formulations. The frequency dependency of the algebraic system resulting from their discretization is also discussed.

\subsection{The interior acoustic problem}

Consider an inviscid compressible fluid comprised in a bounded domain $\Omega$ with boundary $\Gamma$. The boundary $\Gamma$ is divided into two non-overlapping partitions $\Gamma_{n}, \Gamma_{a}$, such that $\Gamma=\Gamma_{n} \cup \Gamma_{a}$. The steady-state acoustic pressure field created by a set of harmonic excitations at wavenumber $k$ is described by the Helmholtz interior boundary value problem

$$
\begin{gathered}
\nabla^{2} p+k^{2} p=-s \quad \text { in } \Omega, \\
\nabla p \cdot \mathbf{n}=-\mathrm{i} \rho \omega v_{n} \quad \text { on } \Gamma_{n}, \\
\nabla p \cdot \mathbf{n}=-\mathrm{i} \rho \omega A_{n} p \quad \text { on } \Gamma_{a},
\end{gathered}
$$

where $k=\omega / c$, with $\omega$, the angular frequency, and $c$, the speed of sound. Eq. (1) is the Helmholtz equation with a source term $s$. In this equation, $p$ denotes the complex amplitude of the pressure representing a time harmonic variation given by $p^{\prime}=\operatorname{Re}\left(p \mathrm{e}^{i \omega t}\right)$. It is assumed that this pressure field represents a small perturbation around an ambient reference state in the fluid. Eqs. (2) and (3) state the Neumann and Robin boundary conditions, respectively, in which $v_{n}, \rho$ and $A_{n}$ represent the normal velocity on the boundary $\Gamma_{n}$, the fluid density and the admittance coefficient value on boundary $\Gamma_{a}$, respectively.

\subsection{The exterior acoustic problem}

Let us now consider the case of an inviscid compressible fluid in an infinite domain $\Omega_{\infty}$. A scattering object with boundary $\Gamma$ is submerged into this fluid. The boundary value problem of radiation and scattering from this obstacle in an unbounded region $\Omega_{\infty}$ consists of Eqs. (1)-(3) together with the Sommerfeld radiation condition

$$
\lim _{|\mathbf{r}| \rightarrow \infty}|\mathbf{r}|\left(\frac{\partial p}{\partial|\mathbf{r}|}-\mathrm{i} k p\right)=0
$$

where $\mathbf{r}$ represents the domain position vector. This condition expresses the fact that no energy can possibly come from infinity, thus guaranteeing the uniqueness of the solution.

In order to render this boundary value problem amenable for finite element discretization, it is necessary to reformulate it into an equivalent bounded one. This is achieved through the introduction of a PML. The PML technique, which was first proposed by Berenger [33], is a popular technique for the simulation of waves in unbounded media due to its effectiveness, simplicity and flexibility [34-36]. The idea is to surround the computational domain by a nonphysical PML medium in which the outgoing waves are absorbed.

The computational domain for modeling exterior Helmholtz problems using PML is shown in Fig. 1. The unbounded domain $\Omega_{\infty}$ is first truncated using some artificial surface $\Gamma_{\text {int }}$ which encloses the scatterer and all the sources. The volume comprised between $\Gamma$ and $\Gamma_{\text {int }}$ defines the physical computational domain denoted $\Omega_{\text {phy }}$, where the solution of the boundary value problem is desired. The volume contained between $\Gamma_{\text {int }}$ and some outer surface $\Gamma_{\text {ext }}$ defines the nonphysical PML region $\Omega_{\mathrm{pml}}=\Omega \backslash \Omega_{\mathrm{phy}}$.

The PML derivation typically relies on a global separable coordinate system. Using Cartesian, spherical or cylindrical based PML definitions and combining them, a wide range of geometries can be efficiently tackled. Still, in some applications, an unnecessarily large free-space region must be discretized between the PML and the object under investigation. Consequently, researchers tried to extend the flexibility of the method by implementing more general formulations. Lassas et al. [37] 


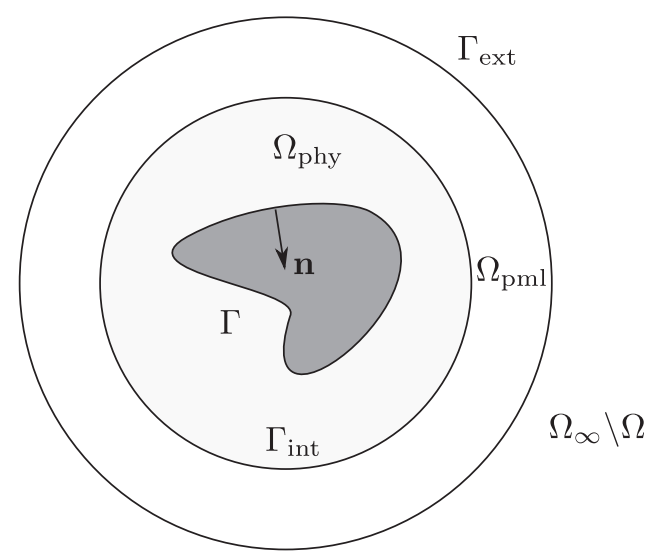

Fig. 1. Computational domain for exterior acoustics applications.

introduced a normal tangential coordinate system based on a parametrization of the boundary. Later on, Zschiedrich et al. [38] developed a specific coordinate system able to cope with any polygonal domains. Recently, a new PML realization was proposed by Ozgun et al. called "locally conformal PML" [23,39], which allows to easily design an arbitrarily shaped convex absorbing region. The originality of the method lies in the fact that the variational formulation is solved directly in the complex space, yielding a conceptually simple scheme which retains most of the regular FEM implementation.

In this paper, the locally conformal PML realization is used. In this approach, each point $P$ in $\Omega_{\mathrm{pml}} \subset \mathbb{R}^{3}$ is mapped onto $\tilde{P}$ in the complex PML region $\Theta \subset \mathbb{C}^{3}$ based on the following complex coordinate transformation [39]:

$$
\tilde{\mathbf{x}}=\mathbf{x}+\frac{1}{\mathrm{i} k} g(\xi) \mathbf{n}(\xi),
$$

where $\mathbf{x} \in \mathbb{R}^{3}$ and $\tilde{\mathbf{x}} \in \mathbb{C}^{3}$ are the position vectors of the points $P$ in real space and $\tilde{P}$ in complex space, respectively. The parameter $\xi \in[0,1]$ determines the local positioning of $P$ inside the layer, the unit vector $\mathbf{n}$ indicates the outward absorption axis and $g(\xi)$ is a monotonically increasing function of $\xi$, typically quadratic, which is referred to as the absorption function. This function vanishes at $\xi=0$ to ensure the continuity of the transformation (5) at the interface $\Gamma_{\text {int }}$ with the physical domain. Note that if a spherical absorbing layer is considered, the transformation (5) is shown to be equivalent to the classical spherical PML transformation [23].

The Helmholtz equation is modified inside the PML medium through the complex coordinate transformation (5) as follows:

$$
\tilde{\nabla}^{2} \tilde{p}(\tilde{\mathbf{x}})+k^{2} \tilde{p}(\tilde{\mathbf{x}})=0 \quad \forall \mathbf{x} \text { in } \Omega_{\mathrm{pml}},
$$

where $\tilde{p}$ is the analytic continuation of the unknown pressure field to complex space and $\tilde{\nabla}$ is the transformed nabla operator which reads

$$
\tilde{\nabla}=\left[\mathbf{J}^{-1}\right]^{T} \cdot \nabla,
$$

with $\mathbf{J}$, the Jacobian matrix of transformation (5) with entries

$$
\mathbf{J}_{[i, j]}=\frac{\partial \tilde{x}_{i}}{\partial x_{j}} .
$$

Inside the physical domain, the standard Helmholtz equation (1) holds and the continuity between the fields $p$ and $\tilde{p}$ is ensured by the following relation:

$$
\tilde{\mathbf{x}}=\mathbf{x} \quad \forall \mathbf{x} \in \Gamma_{\text {int }} .
$$

On the external surface $\Gamma_{\text {ext }}$ a Dirichlet boundary condition is applied

$$
\tilde{p}=0 \quad \forall \mathbf{x} \in \Gamma_{\text {ext }} .
$$

In summary, the Helmholtz exterior boundary value problem consists of Eq. (6) in the PML medium, of Eq. (1) in the physical domain and of the conditions (2), (3) and (9) applied on the boundary $\partial \Omega=\Gamma \cup \Gamma_{\text {ext }}$.

\subsection{Finite element formulation}

For the sake of conciseness, only the weak form associated to the exterior acoustic boundary problem described in Section 2.2 is presented. The expression corresponding to the interior configuration can be easily retained by removing all PML medium contributions. 
The weak form on the PML medium can be obtained using the method of weighted residuals and is given in the complex space as

$$
\int_{\Theta}\left\{\tilde{\nabla} \tilde{q} \cdot \tilde{\nabla} \tilde{p}-k^{2} \tilde{q} \tilde{p}\right\} \mathrm{d} \Theta,
$$

where $\tilde{q}$ is a scalar weight function in the complex space vanishing at $\Gamma_{\text {ext }}$. The weak form (10) can be transformed into the real space coordinates by using Eq. (7)

$$
\left.\int_{\Omega_{\mathrm{pml}}}\left\{\left(\mathbf{J}^{-1}\right]^{T} \cdot \nabla \tilde{q}\right) \cdot\left(\left[\mathbf{J}^{-1}\right]^{T} \cdot \nabla \tilde{p}\right)-k^{2} \tilde{q} \tilde{p}\right\} \operatorname{det}(\mathbf{J}) \mathrm{d} \Omega_{\mathrm{pml}} \cdot
$$

In most PML realizations, the real space form (11) is used. The originality of the locally conformal PML method lies in the fact that it relies directly on the complex residual statement (10). This formulation is solved directly in the complex space, using elements with complex nodal coordinates (for more details, see [23]). After adding the physical domain contribution, the Helmholtz exterior boundary value problem leads to the following integral formulation:

$$
\int_{\Theta}\left\{\tilde{\nabla} \tilde{q} \cdot \tilde{\nabla} \tilde{p}-k^{2} \tilde{q} \tilde{p}\right\} \mathrm{d} \Theta+\int_{\Omega_{\mathrm{phy}}}\left\{\nabla q \cdot \nabla p-k^{2} q p\right\} \mathrm{d} \Omega_{\mathrm{phy}}-\mathrm{i} \omega \int_{\Gamma_{a}} \rho A_{n} q p \mathrm{~d} \Gamma_{a}=\int_{\Omega_{\mathrm{phy}}} q s \mathrm{~d} \Omega_{\mathrm{phy}}+\mathrm{i} \omega \int_{\Gamma_{n}} \rho q v_{n} \mathrm{~d} \Gamma_{n},
$$

for all functions $(p, q)$ continuous, piecewise linear in $\Omega_{\text {phy }}$ and for all functions $(\tilde{p}, \tilde{q})$ continuous, piecewise linear in $\Theta$ and vanishing on $\Gamma_{\text {ext }}$.

\subsection{Algebraic system}

The discretization of the weak form (12) yields the following algebraic system:

$$
\mathbf{Z}(f) \mathbf{p}(f)=\mathbf{b}(f), \quad f=\frac{\omega}{2 \pi},
$$

where $\mathbf{Z}$ is a sparse, square, complex symmetric matrix of size $N, \mathbf{p}$ is the vector containing the nodal pressure unknowns and $\mathbf{b}$ is the right-hand side vector. The size of the system depends on the number of degrees of freedom in the discretization, but it ranges typically from $10^{4}$ to $10^{6}$ for industrially relevant problems. Solving Eq. (13) over a large set of frequencies $F$

$$
F=\left\{f_{1}, f_{2}, \ldots, f_{N_{f}}\right\}, f_{1}=f_{\min }, f_{N_{f}}=f_{\max }
$$

is referred to as a frequency sweep problem. For interior acoustics applications with constant impedance $A_{n}$, the system matrix $\mathbf{Z}$ exhibits a second-order frequency dependency

$$
\mathbf{Z}(f)=\mathbf{K}-(2 \pi f)^{2} \mathbf{M}+\mathrm{i}(2 \pi f) \mathbf{D},
$$

with constant stiffness matrix $\mathbf{K}$, mass matrix $\mathbf{M}$ and damping matrix $\mathbf{D}$. For such algebraic systems and for a constant righthand side, $\mathbf{b}(f)=\mathbf{b}$, Krylov-based algorithms such as SOAR [16] can be used efficiently.

In any other case, such approaches are not directly applicable. For instance, the system arising from the discretization of the Helmholtz-PML formulation is much more complex. In fact, the in-depth analysis provided hereafter reveals that it is rational of high order. Let us consider the frequency dependency of the Jacobian matrix given in Eq. (8). From the transformation (5), the following relation holds:

$$
\mathbf{J}=\mathbf{H}_{1}+\frac{1}{k} \mathbf{H}_{2},
$$

where $\mathbf{H}_{1}, \mathbf{H}_{2}$ are frequency independent. Considering the expression of the integral in Eq. (11), the frequency dependency of the PML entries originates from the contributions of the matrix inverse and of the determinant of the Jacobian $\mathbf{J}$. Consequently, the system matrix $\mathbf{Z}$ arising from computations involving PML exhibits a frequency dependency of rational kind, which cannot be written under the quadratic form in Eq. (15).

The PML elements used in the numerical examples in Section 6 are provided by the commercial software LMS Virtual.Lab Acoustics [40]. Constant-length layers of elements are directly extruded from the triangle mesh outer FEM surface, following the node normals, yielding a structured pentahedral mesh pattern in the PML region (see Fig. 9(b)). In Fig. 2 two different pentahedras are studied, a straight one (Fig. 2(a)), as likely to be extruded from a straight surface with collinear normal at nodes, and a distorted one (Fig. 2(b)), as likely to be extruded from a curved surface with different normal at nodes.

The $6 \times 6$ matrix, denoted as $\mathbf{Z}_{e}$, of each element was computed in symbolic closed form to extract the frequency behavior of its entries. For more details about the elementary matrices computation of the locally conformal PML realization, the reader is referred to [23]. The elementary matrix entries of these two elements were found to be of complexity

$$
\mathbf{Z}_{e_{[i, j]}} \sim \mathcal{O}\left(\frac{P_{5}(f)}{Q_{3}(f)}\right) \text { (straight) and } \mathbf{Z}_{e_{[i, j]}} \sim \mathcal{O}\left(\frac{P_{17}(f)}{Q_{15}(f)}\right) \text { (distorted), }
$$

where $P_{n}$ and $Q_{m}$ are non-zero polynomials of degree at most $n$ and $m$ respectively. Concerning the rational function characteristics, a root finding analysis reveals that all poles lie on the positive imaginary axis, except for the pole at $0+0 \mathrm{i}$, which is shared by all matrix entries. The frequency behavior of the entries $[i, j]=[2,2]$ and $[i, j]=[5,4]$ is shown in Fig. 3(a) for non-dimensional wavenumbers between $k=0$ and $k=10$. Both entries clearly exhibit a pole at $k=0$. For the diagonal entry, the 
a

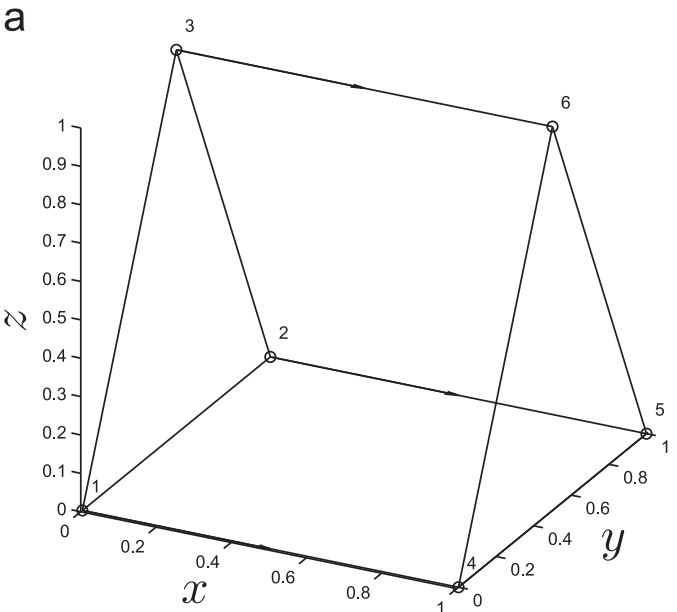

b

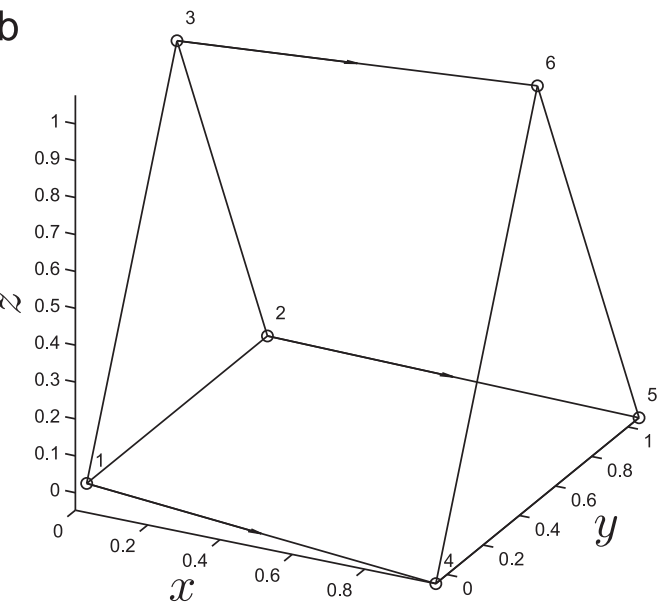

Fig. 2. PML elements considered for the symbolic analysis of the elementary matrix entries. (a) Straight element; (b) Distorted element.
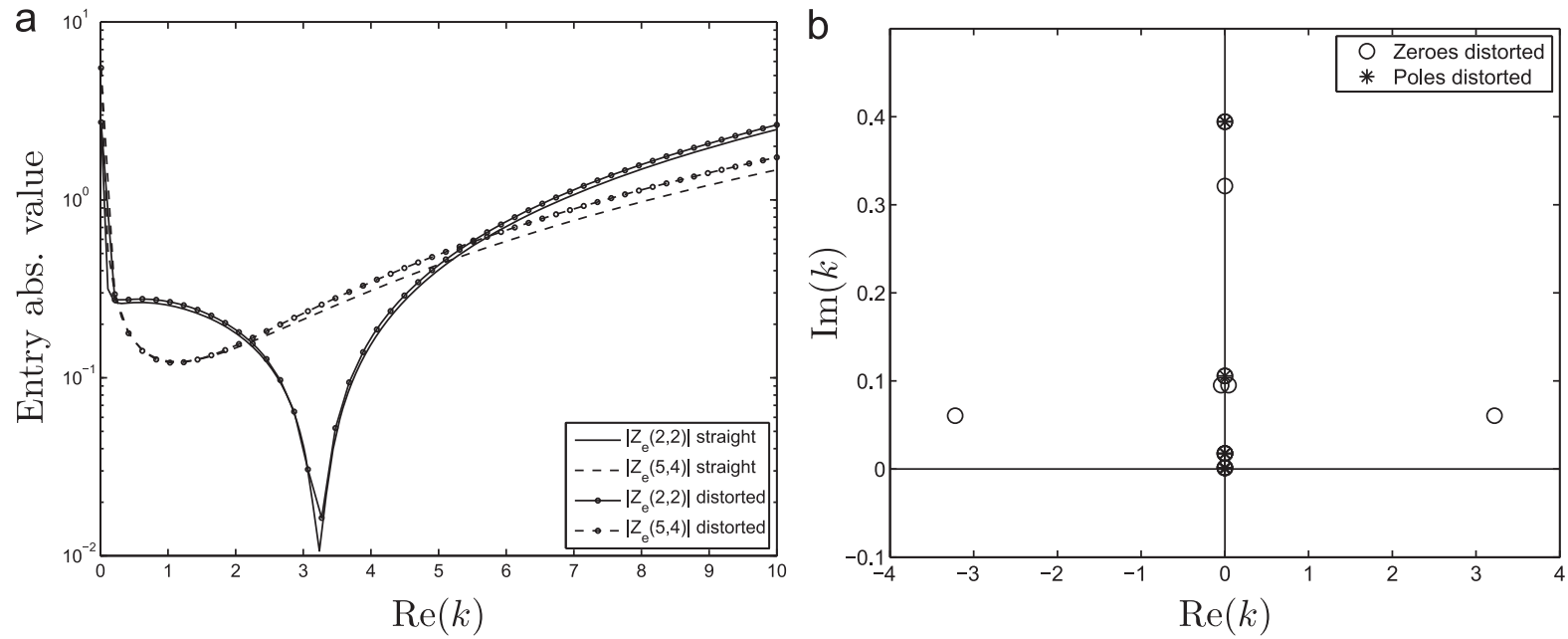

Fig. 3. Analysis of the elementary matrix entries associated to the PML locally conformal elements. (a) Frequency behavior of two entries; (b) Poles and zeroes of the $(2,2)$ entry of the distorted element matrix in the complex plane.

behavior indicates also the presence of a zero between $k=3$ and $k=4$. The poles and zeroes of this diagonal entry for the distorted element are shown in Fig. 3(b). The plot suggests that there are almost pole-zero cancellations, indicating that a rational approximation of order lower that 17 (see Eq. (16)) is able to capture the behavior. This study of the PML entries is relevant for the numerical example in Section 6.

It should be emphasized that the previous analysis only concerns the elementary matrix entries. The PML entries in the global matrix $\mathbf{Z}$ will comprise contributions from all the neighboring elements and, as such, will exhibit a rational behavior of much higher order than those given in Eq. (16). Such systems with complex frequency dependency can only be tackled using Padé based techniques. In the following section, the Well Conditioned Asymptotic Waveform Evaluation is introduced.

\section{Well-conditioned asymptotic waveform evaluation (WCAWE)}

This section introduces the WCAWE algorithm [29]. Using the Taylor coefficients of the system matrix and of the righthand side (RHS), this algorithm computes the subspace of moments in a robust manner by introducing correction factors. In the original paper, the method was applied to systems with polynomial frequency dependency. This paper extends its applicability to systems with rational frequency dependency in some of the entries. Moreover, the original algorithm may suffer from breakdown, so Section 3.2 discusses this possible situation and proposes an improvement.

\subsection{Original algorithm}

This section presents the efficient computation via WCAWE of the subspace of moments used in the Galerkin projection performed to reduce the complexity of the original problem. 
For the time being, assume a single expansion frequency $f_{0}$ (the case of multiple expansion points is treated in Section 5) and denote $f-f_{0}$ by $\sigma$. One can write a Taylor series expansion around $f_{0}$ for the solution vector

$$
\mathbf{p}(f)=\mathbf{p}\left(f_{0}\right)+\mathbf{p}^{\prime}\left(f_{0}\right) \sigma+\mathbf{p}^{\prime \prime}\left(f_{0}\right) \frac{\sigma^{2}}{2 !}+\ldots+\mathbf{p}^{(n)}\left(f_{0}\right) \frac{\sigma^{n}}{n !}+\ldots
$$

where $\mathbf{p}^{(n)}$ denotes the $n$ th-order derivative of $\mathbf{p}$. The derivatives are obtained by differentiating Eq. (13), which yields the following recursive expression:

$$
\mathbf{p}^{(n)}\left(f_{0}\right)=\left.\frac{\partial^{n} \mathbf{p}}{\partial f^{n}}\right|_{f=f_{0}}=\mathbf{Z}\left(\mathbf{f}_{0}\right)^{-1}\left[\left.\frac{\partial^{n} \mathbf{b}}{\partial f^{n}}\right|_{f=f_{0}}-\left.\left.\sum_{i=1}^{n} C_{n}^{i} \frac{\partial^{i} \mathbf{Z}}{\partial f^{i}}\right|_{f=f_{0}} \frac{\partial^{n-i} \mathbf{p}}{\partial f^{n-i}}\right|_{f=f_{0}}\right],
$$

where $C_{n}^{i}=n ! / i !(n-i)$ ! is the binomial coefficient. Introducing the following notations:

$$
\begin{gathered}
\mathbf{w}_{n+1}=\left.\frac{1}{n !} \frac{\partial^{n} \mathbf{p}(f)}{\partial f^{n}}\right|_{f=f_{0}}, \\
\mathbf{Z}_{n}=\left.\frac{1}{n !} \frac{\partial^{n} \mathbf{Z}(f)}{\partial f^{n}}\right|_{f=f_{0}}, \\
\mathbf{b}_{n}=\left.\frac{1}{n !} \frac{\partial^{n} \mathbf{b}(f)}{\partial f^{n}}\right|_{f=f_{0}},
\end{gathered}
$$

and performing rearrangements, ones finds that the moments, namely $\mathbf{w}_{n+1}$, are obtained through the following process:

$$
\begin{gathered}
\mathbf{w}_{1}=\mathbf{Z}_{\mathbf{0}}^{-1} \mathbf{b}_{0} \\
\mathbf{w}_{2}=\mathbf{Z}_{\mathbf{0}}^{-1}\left(\mathbf{b}_{1}-\mathbf{Z}_{1} \mathbf{w}_{1}\right) \\
\vdots \\
\mathbf{w}_{n+1}=\mathbf{Z}_{\mathbf{0}}^{-1}\left[\mathbf{b}_{n}-\sum_{i=1}^{n} \mathbf{Z}_{i} \mathbf{w}_{n-i+1}\right] .
\end{gathered}
$$

Galerkin Asymptotic Waveform Evaluation amounts to forming the moment-matching subspace $\mathbf{W}_{n}=\left[\mathbf{w}_{1} \ldots \mathbf{w}_{n}\right] \in \mathbb{C}^{N \times n}$ and imposing that the residual is perpendicular to $\mathbf{W}_{n}$, yielding the following solution vector:

$$
\mathbf{p}_{n}(f)=\mathbf{W}_{n}\left(\mathbf{W}_{n}^{H} \mathbf{Z}(f) \mathbf{W}_{n}\right)^{-1}\left(\mathbf{W}_{n}^{H} \mathbf{b}(f)\right),
$$

where $(\cdot)^{H}$ stands for the complex conjugate transpose. However, the moments computed using Eq. (21) become linearly dependent very soon (for small values of $n$ ) due to repetitive premultiplication by $\mathbf{Z}_{\mathbf{0}}^{-1}$, causing the vectors to align in the direction of the eigenvector associated to the largest eigenvalue of the matrix $\mathbf{Z}_{\mathbf{0}}^{-1}$. Therefore, the aim of Well Conditioned Asymptotic Waveform Evaluation [29] is to compute this space in an alternative way, which yields a meaningful result even for high $n$. The pseudocode is outlined in Algorithm 1.

\section{Algorithm 1. WCAWE}

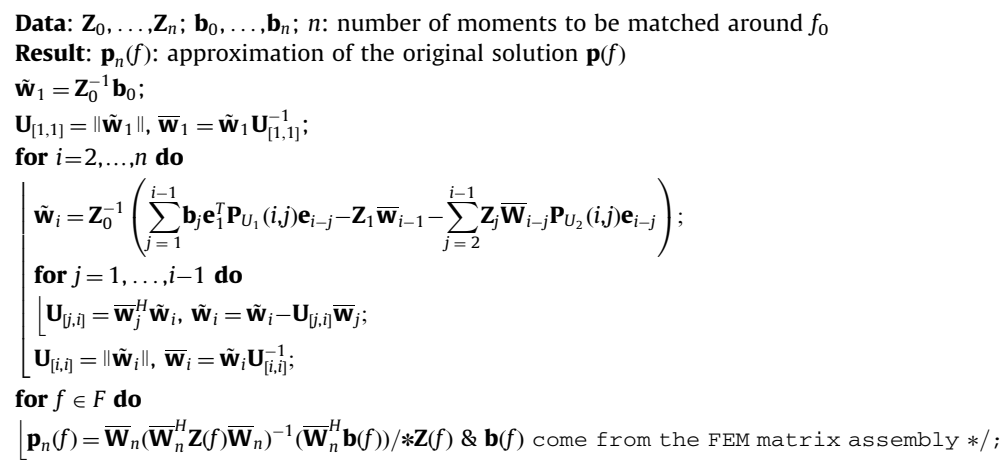

In the following, some notation is clarified:

- $\mathbf{P}_{U_{w}}(i, j)=\prod_{k=w}^{j} \mathbf{U}_{[k:(i-j+k-1), k:(i-j+k-1)]}^{-1}$

- $\mathbf{U}_{[i: j, i: j]}$ denotes the block of the matrix $\mathbf{U}$ from rows $i$ to $j$ and columns $i$ to $j$, 
- $\mathbf{e}_{i}$ is the $i$ th unit vector: all its entries are 0 except the $i$ th, which is 1 , so the operation $\mathbf{e}_{1}^{T} \mathbf{P}_{U_{1}}(i, j) \mathbf{e}_{i-j}$ selects the entry on row 1 and column $i-j$ of the matrix $\mathbf{P}_{U_{1}}(i, j)$, while $\mathbf{P}_{U_{2}}(i, j) \mathbf{e}_{i-j}$ is selecting the $i-j$ column of $\mathbf{P}_{U_{2}}(i, j)$.

\subsection{Breakdown of WCAWE}

The algorithm may break down in some particular situation. Suppose that the Taylor coefficients of the matrix $\mathbf{Z}(f)$ are: $\mathbf{Z}_{0}, \mathbf{Z}_{1}=\mathbf{Z}_{0}, \mathbf{Z}_{2} \neq \mathbf{Z}_{0}$ and those of the right-hand side follow the same pattern: $\mathbf{b}_{0}, \mathbf{b}_{1}=\mathbf{b}_{0}, \mathbf{b}_{2} \neq \mathbf{b}_{0}$. The moments according to Eq. (21) are

$$
\begin{gathered}
\mathbf{w}_{1}=\mathbf{Z}_{\mathbf{0}}^{-1} \mathbf{b}_{0}, \\
\mathbf{w}_{2}=\mathbf{Z}_{\mathbf{0}}^{-1}\left(\mathbf{b}_{1}-\mathbf{Z}_{1} \mathbf{w}_{1}\right)=\mathbf{Z}_{\mathbf{0}}^{-1} \underbrace{(\mathbf{b}_{0}-\underbrace{\mathbf{Z}_{0} \mathbf{Z}_{\mathbf{0}}^{-1}}_{\mathbf{I}} \mathbf{b}_{0})}_{\mathbf{0}}=\mathbf{0}, \\
\mathbf{w}_{3}=\mathbf{Z}_{\mathbf{0}}^{-1}\left[\mathbf{b}_{2}-\mathbf{Z}_{1} \mathbf{w}_{2}-\mathbf{Z}_{2} \mathbf{w}_{1}\right]=\mathbf{Z}_{\mathbf{0}}^{-1}\left[\mathbf{b}_{2}-\mathbf{Z}_{2} \mathbf{Z}_{\mathbf{0}}^{-1} \mathbf{b}_{0}\right] \neq \mathbf{0} .
\end{gathered}
$$

Applying the WCAWE process, described in Algorithm 1, in double precision for this case yields the following:

1. $\tilde{\mathbf{w}}_{1}=\mathbf{Z}_{0}^{-1} \mathbf{b}_{0}, \mathbf{U}_{[1,1]}=\left\|\tilde{\mathbf{w}}_{1}\right\| \Rightarrow \overline{\mathbf{w}}_{1}=\tilde{\mathbf{w}}_{1} \mathbf{U}_{[1,1]}^{-1}$.

2. $\tilde{\mathbf{w}}_{2}=\mathbf{Z}_{0}^{-1}\left(\mathbf{b}_{1} \mathbf{e}_{1}^{T} \mathbf{P}_{U_{1}}(2,1) \mathbf{e}_{1}-\mathbf{Z}_{1} \overline{\mathbf{w}}_{1}\right)=\epsilon$, where $\epsilon$ is a vector with entries below machine precision.

3. $\mathbf{U}_{[1,2]}=\overline{\mathbf{w}}_{1}^{H} \tilde{\mathbf{w}}_{2}=\epsilon \Rightarrow \tilde{\mathbf{w}}_{2}=\tilde{\mathbf{w}}_{2}-\mathbf{U}_{[1,2]} \overline{\mathbf{w}}_{1}=\epsilon$.

4. $\mathbf{U}_{[2,2]}=\left\|\tilde{\mathbf{w}}_{2}\right\|=\epsilon \Rightarrow \overline{\mathbf{w}}_{2}=\tilde{\mathbf{w}}_{2} \mathbf{U}_{[2,2]}^{-1}=\epsilon / \epsilon$. Note that this is non-zero in finite precision, since it is obtained by dividing two small quantities. At this point, breakdown has occurred since $\overline{\mathbf{w}}_{2}$ should be $\mathbf{0}$, or close to machine precision. Therefore, by adding this moment to the space and continuing with the recursive procedure, the following moments are no longer matched.

As inverses of blocks of the upper triangular matrix $\mathbf{U}$ are used in the WCAWE algorithm (the $\mathbf{P}_{U_{w}}(i, j)$ quantities), breakdown will occur when a diagonal entry is below machine precision. This happens when $\tilde{\mathbf{w}}_{i}$ in line 4 of Algorithm 1 is either 0 or a linear combination of vectors already in the subspace. In both cases, the entry $\mathbf{U}_{[i, i]}$ is 0 (or below machine precision), so the modified Gram-Schmidt process fails. The proposed remedy is to set $\mathbf{U}_{[i, i]}$ to 1 . Note that [29] point out that there are no constraints in choosing the coefficients in the matrix $\mathbf{U}$, so changing the value of the $\mathbf{U}_{[i, i]}$ entry from 0 to 1 avoids breakdown, but still ensures that the moment matching property holds. However, this remedy yields an unnecessary column in $\overline{\mathbf{W}}_{n}$, since the $\tilde{\mathbf{w}}_{i}$ moment, which is 0 or linearly dependent on the previous moments, is kept in the projection subspace. Therefore, when computing the approximation, the projection should be done on the subspace formed by the linearly independent moments. This can be computed via a rank-revealing singular value decomposition (SVD) or QR decomposition or by saving the indices of the useful moments. The latter approach is discussed in Algorithm 2.

Algorithm 2. WCAWEnobreakdown

Data: $\mathbf{Z}_{0}, \ldots, \mathbf{Z}_{n} ; \mathbf{b}_{0}, \ldots, \mathbf{b}_{n} ; n$ : number of moments to be matched around $f_{0}$

Result: $\mathbf{p}_{n}(f)$ : approximation of the original solution $\mathbf{p}(f)$

$\tilde{\mathbf{w}}_{1}=\mathbf{Z}_{0}^{-1} \mathbf{b}_{0}$;

$\mathbf{U}_{[1,1]}=\left\|\tilde{\mathbf{w}}_{1}\right\|, \overline{\mathbf{w}}_{1}=\tilde{\mathbf{w}}_{1} \mathbf{U}_{[1,1]}^{-1}$;

$k=1, \hat{\mathbf{w}}_{1}=\overline{\mathbf{w}}_{1} /{ }^{*}$ first 1 inearly independent moment ${ }^{*} /$

for $i=2, \ldots, n$ do

$\mid \begin{aligned} & \tilde{\mathbf{w}}_{i}=\mathbf{Z}_{0}^{-1}\left(\sum_{j=1}^{i-1} \mathbf{b}_{j} \mathbf{e}_{1}^{T} \mathbf{P}_{U_{1}}(i, j) \mathbf{e}_{i-j}-\mathbf{Z}_{1} \overline{\mathbf{w}}_{i-1}-\sum_{j=2}^{i-1} \mathbf{Z}_{j} \overline{\mathbf{W}}_{i-j} \mathbf{P}_{U_{2}}(i, j) \mathbf{e}_{i-j}\right) \\ & \text { for } j=1, \ldots, i-1 \text { do } \\ & \mid \mathbf{U}_{[j, i]}=\overline{\mathbf{w}}_{j}^{H} \tilde{\mathbf{w}}_{i}, \tilde{\mathbf{w}}_{i}=\tilde{\mathbf{w}}_{i}-\mathbf{U}_{[j, i]} \overline{\mathbf{w}}_{j} ; \\ & \mathbf{U}_{[i, i]}=\left\|\tilde{\mathbf{w}}_{i}\right\| ; \\ & \text { if } \mathbf{U}_{[i, i]}<\epsilon \text { then } \\ & \mid \begin{array}{l}\mathbf{U}_{[i, i]}=1, \overline{\mathbf{w}}_{i}=\tilde{\mathbf{w}}_{i} \mathbf{U}_{[i, i]}^{-1} ; \\ \text { else }\end{array} \\ & \mid \begin{array}{l}\overline{\mathbf{w}}_{i}=\tilde{\mathbf{w}}_{i} \mathbf{U}_{[i, i]}^{-1} ; \\ k=k+1, \hat{\mathbf{w}}_{k}=\overline{\mathbf{w}}_{i} / * \text { addlinearlyindependentmoment } * /\end{array}\end{aligned}$

for $f \in F$ do

$\mid \mathbf{p}_{q}(f)=\hat{\mathbf{W}}_{k}\left(\hat{\mathbf{W}}_{k}^{H} \mathbf{Z}(f) \hat{\mathbf{W}}_{k}\right)^{-1}\left(\hat{\mathbf{W}}_{k}^{H} \mathbf{b}(f)\right)$

Algorithm 2 assumes that the first moment is not 0 and therefore has a non-zero norm (see line 2 ). This is a valid assumption since the first moment is simply the response at the expansion frequency $f_{0}$. 


\section{Computing the Taylor coefficients}

Eq. (18) involves the derivatives of the matrix $\mathbf{Z}$ and of the right-hand side $\mathbf{b}$ with respect to frequency. These are not known, as $\mathbf{Z}$ and $\mathbf{b}$ are not analytical functions of frequency, but rather only assembled at discrete frequency values in the desired range. Hence, this section outlines the two main steps related to computing the Taylor coefficients given in Eqs. (19) and (20):

- constructing polynomial or rational interpolating functions for the entries of $\mathbf{Z}$ and $\mathbf{b}$ from the assembled matrices at the desired frequencies (Section 4.1).

- performing the differentiation with respect to frequency of the polynomial or rational interpolating functions (Section 4.2).

Some notation is first introduced. $S_{z}$ denotes the set of non-zero entries of the sparse complex matrix $\mathbf{Z}(f)$ and $N_{z}$ denotes the cardinality of this set. Similarly $S_{b}$ denotes the set of non-zero entries of the sparse vector $\mathbf{b}(f)$ and $N_{b}$, its cardinality. The sparsity patterns $S_{z}$ and $S_{b}$ are assumed to be frequency independent. Within $S_{z}$ and $S_{b}$, the frequency dependencies may be very different from one entry to another. Section 4.1 describes the fitting approach used to identify polynomial or rational functions of appropriate orders for the non-zero matrix entries with complicated frequency behavior. Most matrix entries exhibit a simple frequency dependency (typically quadratic) and a polynomial fit suffices to model them.

\subsection{Interpolation approach}

Denoting by $x_{k}$, the value of some entry $x$ in the sparsity pattern of the matrix $\mathbf{Z}$ or the vector $\mathbf{b}$ at frequency $f_{k}$, obtained from a standard assembly procedure, an approximation $\hat{x}(f)$ is sought, either as a polynomial

$$
\hat{x}(f)=\sum_{h=0}^{m} \alpha_{h} f^{h} \quad \text { for } x \in S_{z} \text { or } x \in S_{b},
$$

or as a rational function.

$$
\hat{x}(f)=\frac{a(f)}{b(f)}=\frac{\sum_{h=0}^{m} \alpha_{h} f^{h}}{\sum_{l=0}^{m} \beta_{l} f^{l}} \quad \text { for } x \in S_{z} \text { or } x \in S_{b} .
$$

The choice of a rational function is motivated by the fact that entries associated with certain properties and/or boundary conditions exhibit singular frequency dependencies (see Section 2.4) which cannot be captured via polynomial interpolation. This interpolation procedure aims at finding the optimal order of the approximation and the associated coefficients $\alpha_{h}, \forall h=0, \ldots, m$ or $\alpha_{h}, \beta_{l}, \forall h, l=0, \ldots, m$.

For polynomial interpolation, computing the coefficients amounts to solving a least-squares system $\mathbf{V}_{m} \boldsymbol{\alpha}=\mathbf{x}$ with

$$
\mathbf{V}_{m}=\left[\begin{array}{ccccc}
1 & f_{1} & f_{1}^{2} & \ldots & f_{1}^{m} \\
1 & f_{2} & f_{2}^{2} & \ldots & f_{2}^{m} \\
\vdots & \vdots & \vdots & \ddots & \vdots \\
1 & f_{N_{f}} & f_{N_{f}}^{2} & \ldots & f_{N_{f}}^{m}
\end{array}\right], \quad \boldsymbol{\alpha}=\left[\begin{array}{c}
\alpha_{0} \\
\alpha_{1} \\
\vdots \\
\alpha_{m}
\end{array}\right], \quad \mathbf{x}=\left[\begin{array}{c}
x_{1} \\
x_{2} \\
\vdots \\
x_{N_{f}}
\end{array}\right],
$$

where $\mathbf{V}_{m}$ denotes the Vandermonde matrix and $m$ is the interpolation order.

For rational interpolation, after multiplying by the denominator and bringing everything on one side, a similar system can be set up: $\left.\left.\left[\mathbf{V}_{m}-\operatorname{diag}(\mathbf{x}) \mathbf{V}_{m}\right]\right]_{\beta}^{\alpha}\right]=\mathbf{0}$. The vector of coefficients is computed as the null space of the tall matrix $\left[\mathbf{V}_{m}-\operatorname{diag}(\mathbf{x}) \mathbf{V}_{m}\right]$.

\subsubsection{Chebyshev points}

The frequency samples used to set up the rows of the Vandermonde matrix in Eq. (24) are user-defined, and typically equidistant. However, as indicated in [41], using equispaced samples prevents convergence of an $m$-point polynomial interpolant to a function $f$ on $[-1,1]$, as $m \rightarrow \infty$, even if $f$ is analytic. This is due to the Runge phenomenon, which causes oscillations near the endpoints. Even for functions for which convergence should take place in theory, this ill-conditioning causes divergence in floating point arithmetic. On the other hand, polynomial interpolants in Chebyshev points converge for analytic functions. Chebyshev nodes over the interval $[a, b]$ are defined as

$$
\frac{a+b}{2}+\frac{b-a}{2} \cos \left(\frac{2 i-1}{2 m} \pi\right), \quad i=1, \ldots, m .
$$

These points are generated in the interval $\left[f_{\min }, f_{\max }\right]$ and used for setting up the Vandermonde matrix in Eq. (24). Chebyshev points are not included in the set $F$ at which the solution is required and therefore this approach requires extra assembling of the system matrix and the right-hand side. Nevertheless, numerical experiments indicate that this extra cost is compensated by the better fitting accuracy, which leads to more accurate moments, eventually leading to gains in accuracy. 


\subsubsection{Implementation aspects}

The basic idea has been described in the previous section, but implementing it in a straightforward manner would lead to an inefficient algorithm. Therefore, this section discusses various implementation aspects.

To determine the interpolation order $m$, one can loop over the possible orders, starting from 2 until some maximum order, e.g., 8, and stop when polynomial interpolation yields acceptable errors. If this was not the case, one can use a similar loop to test which order is appropriate for rational interpolation. Instead of using an error criterion, this paper employs a test for stagnation. If the errors for two consecutive polynomial orders are in the same order of magnitude, the rest of the orders are no longer tested and rational interpolation is used. If stagnation occurs in rational interpolation as well, the polynomial or rational model which yields the lowest error is retained.

Another implementation aspect relates to the number of Chebyshev points used to set up the rows of the Vandermonde matrix in Eq. (24): rather than employing $N_{f}$, we use a percentage $p$ percent (typically $p=5$ or 10). The motivation is that solving the linear system to determine the fitting coefficients can become computationally prohibitive for a large number of points.

The procedure described above is feasible for the right-hand side vector. However, applying it to all entries in the sparsity pattern of the system matrix $\mathbf{Z}(f)$ is very expensive, since $N_{z}$ can reach $10^{7}-10^{8}$ for large-scale systems. Therefore, an exploratory phase, in which the above procedure is applied for the diagonal entries of $\mathbf{Z}(f)$, followed by a final phase, in which the interpolation order for the off-diagonal entries is estimated based on those of the diagonal entries, was implemented. The appropriate kind and order of interpolation for an off-diagonal entry $\mathbf{Z}_{[i, j]}$ is determined as:

- polynomial of the minimum order between those of $\mathbf{Z}_{[i, i]}$ and $\mathbf{Z}_{[j, j]}$, if both entries are polynomial functions; if this yields unsatisfactory results, the maximum order is used;

- rational of the minimum order between those of $\mathbf{Z}_{[i, i]}$ and $\mathbf{Z}_{[j, j]}$, if both entries are rational; if this yields good results, to avoid overfitting, lower orders are also tested and the one with the smallest errors is stored; if the minimum order was unsatisfactory, a rational model of the maximum order is employed;

- polynomial of the minimum order between those of $\mathbf{Z}_{[i, i]}$ and $\mathbf{Z}_{[j, j]}$, if one entry is polynomial and the other is rational; this situation occurs at the boundary of the physical and the PML domains, in which case the physical node exhibits a polynomial behavior, while the PML node is interpolated by a rational function.

It is worth emphasizing that the proposed system identification process is purely algebraic, as it only requires the system matrix and right-hand side entries assembled at the desired frequencies. Provided that all entries can be written under the form (22) or (23) for moderate orders, no specific a priori knowledge of the boundary value problem at hand is needed and the proposed approach will closely match the original moments. On the other hand, if some of the matrix or right-hand side entries exhibit a more complex frequency dependency (for locally conformal PML applications for instance) the system entries can only be approximated through a fitting procedure. If this fitting procedure fails, based on some predefined deviation criterion, the user has the possibility to split the initial frequency sweep problem into smaller ones. When the system identification process is judged satisfactory enough on each subproblem, the FFS algorithm can be applied.

\subsection{Computing the derivatives}

Recall that $\mathbf{Z}(f)$ and $\mathbf{b}(f)$ are only known at a discrete number of frequency samples. Up to this point, the construction of a continuous model in the frequency, which can be differentiated, was discussed. This section discusses the differentiation process.

For entries interpolated by polynomials, computing derivatives is rather straightforward. Suppose $\hat{x}(f)$ is the continuous polynomial obtained from fitting entry $i$ (see Eq. (22)). The first derivative is simply $\hat{x}^{\prime}(f)=\sum_{h=0}^{m-1}(h+1) \alpha_{h+1} f^{h}$, the second one is $\hat{x}^{\prime \prime}(f)=\sum_{h=0}^{m-2}(h+1)(h+2) \alpha_{h+2} f^{h}$, and so on, until the $m+1$ derivative, which is 0 .

On the other hand, for entries interpolated by rational functions, computing derivatives is more involved. Suppose $\hat{x}(f)$ is the continuous rational function obtained from fitting entry $i$ (see Eq. (23)). The first step amounts to computing the derivatives of the numerator and denominator polynomials, respectively, as outlined in the previous paragraph. Next, the following recursive formula can be applied (the quotient formula):

$$
\hat{x}^{(h)}(f)=\frac{a^{(h)}(f)-\sum_{l=0}^{h-1} C_{h}^{l} \hat{x}^{(l)}(f) b^{(h-l)}(f)}{b(f)} .
$$

\section{Multiple expansion points strategy}

To obtain accurate solutions over a wide frequency range, a multi-frequency strategy must be coupled with the Padé approximation to allow for an efficient fast frequency sweep algorithm. This strategy can be applied in two ways. The classical approach is to use rational-like subspace methods [42,43], where a single subspace is built using vectors from all expansion points. Another approach is to simply store the approximate solution $\mathbf{p}_{n}(f)$ for the frequencies close to the expansion point where the approximation is accurate and build a new moments subspace when considering a new expansion point [10].

When applying Padé approximations, one of the main issues is where to place the expansion points. For systems with known dynamic behavior (i.e. known natural frequencies), there exists a clear connection between the location of the 
interpolation points and the convergence behavior of the approximation [42]. However, in practice, the behavior of the original system is rarely known a priori and the choice may only be motivated through experience.

Moreover, the size of the subspace built around each expansion frequency is another parameter which influences the quality of the approximation. Two different strategies may be chosen: an order-adaptive approach, where the subspace order is increased with the distance to the expansion frequency, and a constant-order approach.

All these issues can be addressed through a proper sequential algorithm. The Adaptive Windowing Algorithm (AWA) of Tuck-Lee et al. [10] provides an intuitive and simple framework to generate non-overlapping frequency windows which span a given frequency range. A "window" refers to the frequency range covered successfully (with respect to a predefined error estimator) by the approximation from one expansion point. The expansion frequencies are simply placed at the center (in a linear or logarithmic sense) of the frequency window. This algorithm is therefore not of rational kind, in the sense that the moments subspaces are not combined. Furthermore, AWA is an order adaptive algorithm as, starting from an initial subspace order $n_{\min }$, the increase in order is achieved through a comparison between two approximations $\mathbf{p}_{n_{k}}$ and $\mathbf{p}_{n_{k+1}}$, where $n_{k+1}=n_{k}+\Delta n$ and $\Delta n$ is a positive integer. If the difference is below the desired tolerance, the solution $\mathbf{p}_{n_{k}}$ is considered to have converged within that particular window. The process stops when $n_{k}$ exceeds some predefined maximum subspace order $n_{\max }$, in which case one or two new windows are created according to the remaining frequencies where the approximation was poor. The procedure continues until both edges of the initial frequency range are reached.

In this paper, a slightly different version of the AWA algorithm is implemented. Rather than checking the convergence of two consecutive subspace orders, the error estimator driving the windowing algorithm is simply defined as the normalized residual

$$
r_{n_{k}}\left(f_{i}\right)=\frac{\left\|\mathbf{Z}\left(f_{i}\right) \mathbf{p}_{n_{k}}\left(f_{i}\right)-\mathbf{b}\left(f_{i}\right)\right\|}{\left\|\mathbf{b}\left(f_{i}\right)\right\|} \text { for } i=1, \ldots, N_{f} .
$$

This is compared to a user-defined accuracy value $\epsilon_{\text {tol }}$ at frequencies $f_{i}$ above and below the expansion frequency. If it is below $\epsilon_{\mathrm{tol}}$, the approximation $\mathbf{p}_{n}\left(f_{i}\right)$ is stored for that particular frequency, otherwise the subspace is increased by $\Delta n$, until it reaches $n_{\max }$ or the bounds of the initial frequency bandwidth.

The AWA scheme is depicted in Fig. 4. It should be noted that some frequency windows (i.e. frequency windows 3 and 4) can be sufficiently represented by a small subspace order, whereas in the other frequency windows the maximum number of moments are required to accurately represent the solution.

In summary, the FFS approach proposed in the paper consists of the following steps. It begins with the system identification process described in Section 4.1. Once this step is completed, the windowing algorithm defines in an iterative manner that bounds the current window and the position of the expansion frequency. At each expansion frequency, also referred to as master frequency, the following four steps are performed:

- Taylor coefficients: computed according to Section 4.2;

- System assembly: assembly of the FE system matrix and right-hand side vector;

- Matrix factorization: factorization of the system matrix;

- Subspace computation: application of WCAWE, described in Algorithm 2 lines 1-13.

For all the other frequencies inside the window, also referred to as slave frequencies, three main operations are performed:

- System assembly: assembly of the FE system matrix and right-hand side vector;

- Reduced system solving: projection of the original system onto the subspace and solving the reduced system as shown in line 15 of Algorithm 2;

- Residual comparison: computation of the residual using Eq. (26) and comparison with the user defined criterion $\epsilon_{\text {tol }}$.

The timings of the different processes will be indicated in the numerical examples.

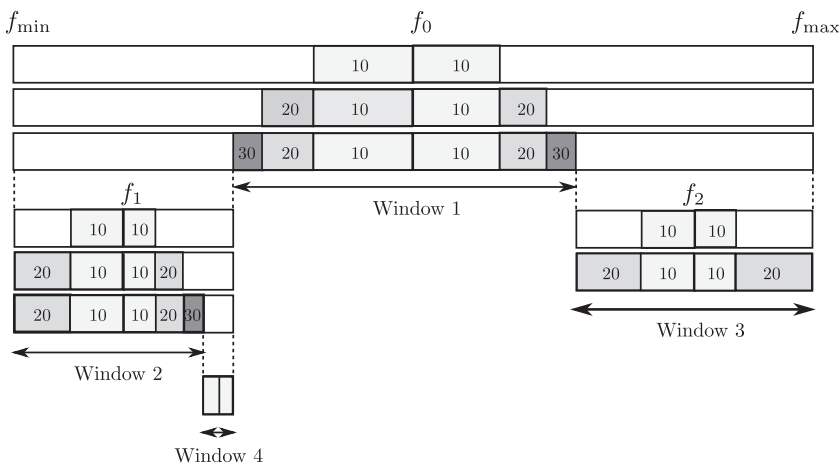

Fig. 4. Illustration of the AWA scheme considering initial subspace order $n_{\min }=10$, maximum subspace order $n_{\max }=30$ and $\Delta n=10$. 


\section{Numerical examples}

This section discusses several numerical examples validating the proposed approach. In Section 6.1, a typical interior acoustics problem is investigated. The proposed WCAWE implementation is first benchmarked against SOAR on a secondorder system with constant admittance. For the second test case, the performance of the FFS is assessed on a large scale non-second-order system with realistic acoustic treatment.

Section 6.2 is devoted to an exterior acoustics problem involving PML. The first test case consists of a sphere with a vibrating cap radiating in free field. An analytical solution is provided to assess the accuracy of the discrete model. In a second test case, a problem involving a non-spherical PML is investigated. The efficiency of the FFS algorithm is judged in terms of both time and accuracy.

\subsection{Interior acoustics problem}

The interior acoustics test case illustrated in Fig. 5 represents a simplified Finite Element (FE) model of a car cavity. The coarse and refined meshes used for the computations are shown in Fig. 5(a) and (b) respectively. The three dimensions of the model as well as the different boundary condition sets are shown in Fig. 5(c). To model some acoustic treatments, admittance boundary conditions are applied on the headliner and on the carpet list of faces. The engine loading is represented either by a point source or by a normal velocity applied on the firewall. The pressure field inside the car cavity is governed by the interior Helmholtz boundary value problem presented in Section 2.1.

\subsubsection{WCAWE vs SOAR}

Let us first consider the case of a constant admittance $A_{n}(f)=A_{n}$ and a constant right-hand side $\mathbf{b}(f)=\mathbf{b}$, as obtained when applying a point source. In this case, the system matrix arising from the FE discretization can be written under the quadratic form given in Eq. (15) and Krylov subspace techniques can be employed. The SOAR algorithm introduced in [16] was implemented in the Matlab programming language for benchmarking against WCAWE.

The coarse mesh used for the analysis depicted in Fig. 5(a) contains 1002 nodes and 1615 tetrahedral elements. The sound speed and fluid density are respectively $c=340 \mathrm{~m} / \mathrm{s}$ and $\rho=1.225 \mathrm{~kg} / \mathrm{m}^{3}$. A constant normalized admittance $\overline{A_{n}}=A_{n} \times \rho c=6.8 \times 10^{-3}+6.9 \times 10^{-2} \mathrm{i}$ is applied on the headliner and carpet set of faces. A constant unitary source of the form $s\left(\mathbf{x}_{\mathbf{s}}\right)=-4 \pi \delta\left(\mathbf{x}-\mathbf{x}_{\mathbf{s}}\right)$ is applied at $\mathbf{x}_{\mathbf{s}}=[0.5,0.45,0.9]$. The frequency range of interest is $f_{\min }=10 \mathrm{~Hz}$ up to $f_{\max }=200 \mathrm{~Hz}$, with increments of $1 \mathrm{~Hz}$. In this test case, the goal is to compare the WCAWE and the SOAR approximations from a single subspace with no adaptive windowing. The single expansion frequency is chosen in the middle of the frequency range at $f_{0}=105 \mathrm{~Hz}$. Furthermore, the system identification process described in Section 4 is not applied as the system is assumed to be of second-order kind and the Taylor coefficients are automatically retrieved from Eq. (15).
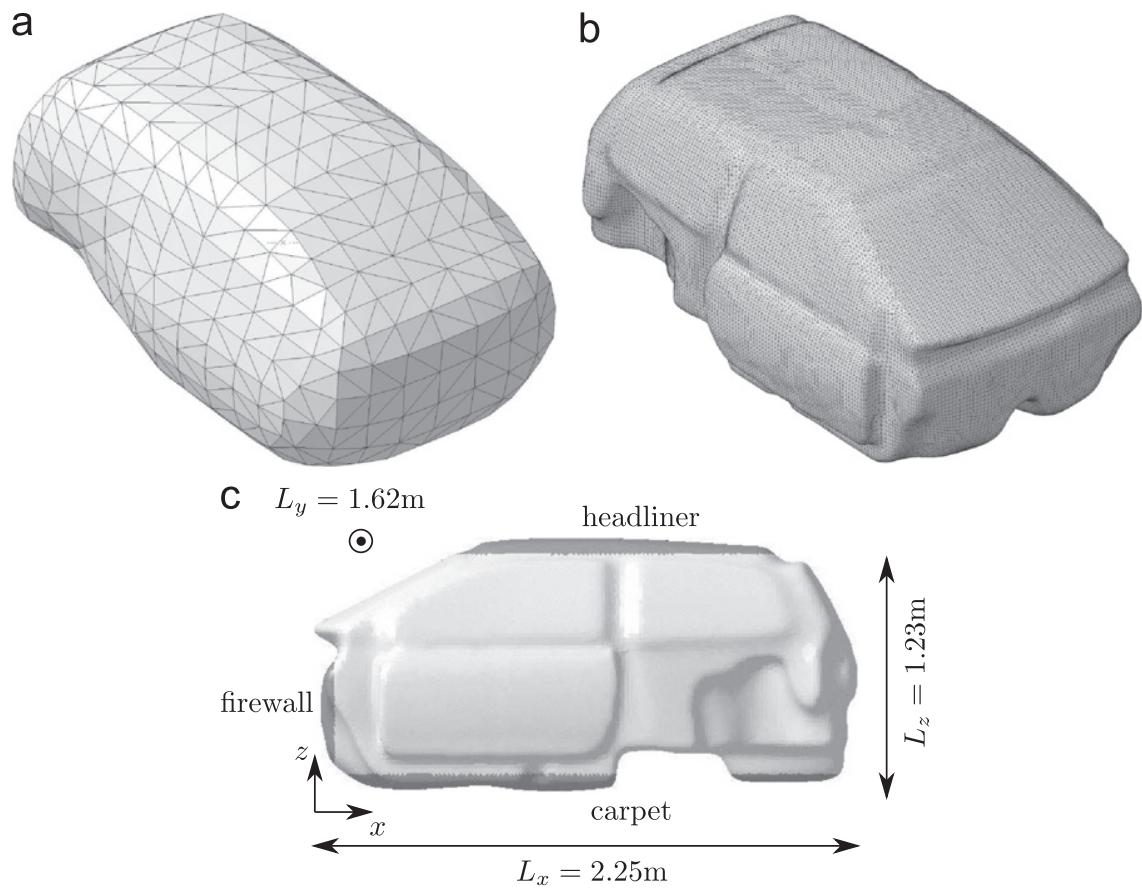

Fig. 5. Finite element mesh and geometry of the car cavity model. (a) Coarse mesh, 1002 nodes; (b) Refined mesh, 472696 nodes; (c) Dimensions of the model and boundary conditions sets. 

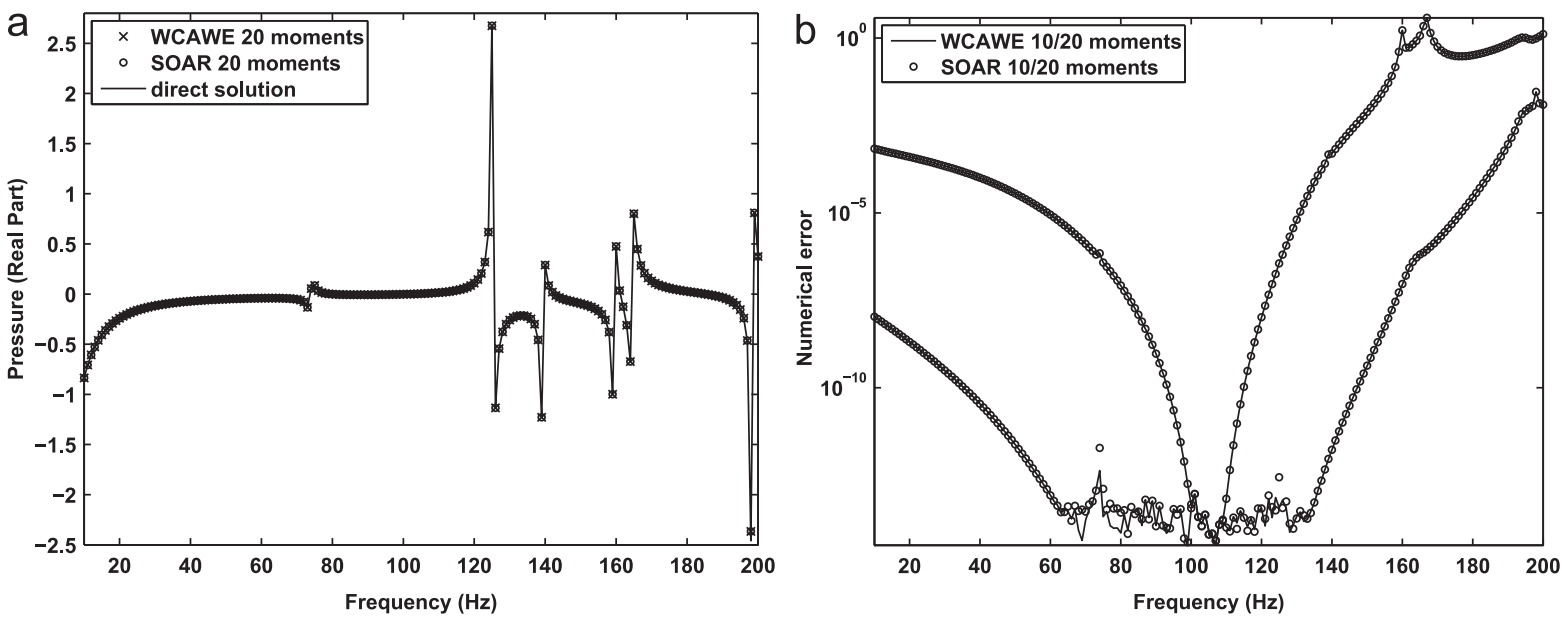

Fig. 6. Comparison of the SOAR and WCAWE algorithms on the coarse mesh car cavity model. (a) Frequency response (entry 261); (b) Normalized error at all entries.

In Fig. 6(a), the real part of the response for entry 261 of the solution vector $\mathbf{p}$ is compared to two approximations obtained by matching $n=20$ moments: the first obtained with WCAWE, and the second with SOAR. Both approximations are in very close agreement as they match the original behavior in the entire frequency window, even close to resonances. In Fig. 6(b), the normalized numerical error of the solution vector $\mathbf{p}(f)$ is plotted for the two methods. To study the effect of the size of the moments subspace, results are shown for $n=10$ and 20. These results confirm that WCAWE and SOAR yield similar approximations. The convergence with respect to the subspace size $n$ is also satisfactory. When increasing from 10 to 20 moments, the numerical error is significantly reduced, which expands the frequency range of validity of the two approximations.

\subsubsection{Large scale test case}

The proposed FFS approach combining the system identification, the WCAWE algorithm and the AWA procedure is now tested on a non-second-order large scale system with realistic acoustic treatment description. The refined mesh used for the computation is depicted in Fig. 5(b). It contains 472696 nodes and 2751336 tetrahedral elements. Using a rule of thumb of 10 grid points per wavelength, the mesh upper frequency limit is $1200 \mathrm{~Hz}$. The normalized admittances of the headliner and the carpet are assumed to be cubic functions of frequency

$$
\begin{gathered}
\overline{A_{n}}=(0.025 \mathrm{i}+0.034 \mathrm{i}) \bar{f}+(0.25+0.67 \mathrm{i}) \bar{f}^{2}-(0.15+0.60 \mathrm{i}) \bar{f}^{3} \quad \text { (headliner), } \\
\overline{A_{n}}=(0.08 \mathrm{i}+0.20 \mathrm{i}) \bar{f}+(1.72+1.77 \mathrm{i}) \bar{f}^{2}-(1.13+1.92 \mathrm{i}) \bar{f}^{3} \quad \text { (carpet), }
\end{gathered}
$$

with $\bar{f}=f / 2000$, hence yielding an algebraic system $\mathbf{Z}(f)$ with fourth-order polynomial frequency dependency. The admittance values are plotted in the range $[10,2000] \mathrm{Hz}$ in Fig. 7.

To represent the engine loading, a constant velocity $v_{n}=1 \times 10^{-4} \mathrm{~m} / \mathrm{s}$ is applied on the firewall, yielding a first-order polynomial frequency dependent right-hand side $\mathbf{b}(f)$. The WCAWE algorithm was implemented in the FORTRAN 90 computing language within the LMS vibro-acoustic solver. Computations were performed on a PC system with $24 \mathrm{~Gb}$ of RAM and $2.80 \mathrm{GHz}$ CPU speed. For all sparse system matrix factorizations (including for the direct approach), the state-ofthe-art MUltifrontal Massively Parallel sparse direct Solver (MUMPS) [44] was used.

The frequency response is required over the range $[20,1200] \mathrm{Hz}$ with a $1 \mathrm{~Hz}$ increment (1181 individual frequencies). The corresponding non-dimensional Helmholtz number ranges between $k D=0.6$ and $k D=36$, where the typical model dimension is $D=1.62 \mathrm{~m}$. The FFS algorithm begins with the system identification presented in Section 4.1. The fitting procedure is applied on a set of 59 Chebyshev points generated in the range $[20,1200] \mathrm{Hz}$. The outcome of the exploratory fitting phase performed on the 472696 diagonal entries is the following:

- 98.15 percent of the entries are interpolated by a polynomial of order 2 .

- 1.85 percent of the entries are interpolated by a polynomial of order 4 .

The 3260451 off-diagonal entries are then interpolated with polynomial orders 2 and 4 , according to the results of the exploratory phase. The outcome of the final fitting procedure is as follows:

- 99.21 percent of the entries are interpolated by a polynomial of order 2 .

- 0.79 percent of the entries are interpolated by a polynomial of order 4 . 


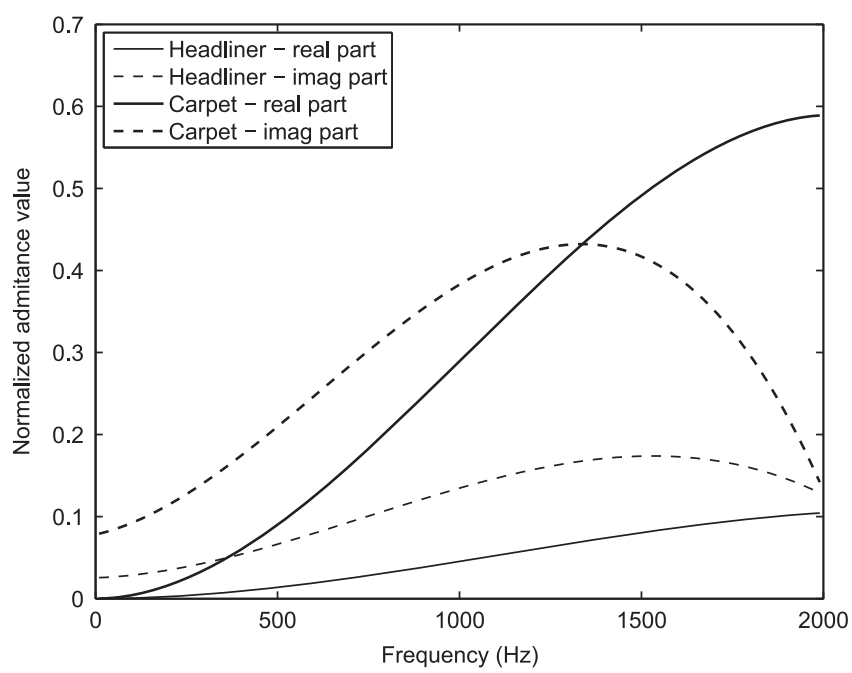

Fig. 7. Cubic normalized admittance values used for the headliner (thin line) and the carpet (bold line) of the car cavity.

In terms of computational cost, the exploratory and final fitting phases are performed in $12 \mathrm{~s}$ and $26 \mathrm{~s}$, respectively. Fitting errors are quantified by measuring the maximum and the mean entry deviations

$$
d_{\max }\left(f_{k}\right)=\max _{x \in S_{z}, S_{b}} \frac{\left|\hat{x}\left(f_{k}\right)-x_{k}\right|}{\left|x_{k}\right|},
$$

and

$$
d_{\text {mean }}\left(f_{k}\right)=\left(\sum_{\forall x \in S_{z}, S_{b}} \frac{\left|\hat{x}\left(f_{k}\right)-x_{k}\right|}{\left|x_{k}\right|}\right) \times \frac{1}{N_{z}+N_{b}}
$$

at each frequency $f_{k}$ in the set $F$. In this particular test case, the maximum and mean matrix entry deviations do not vary significantly with frequency and are of the following orders of magnitude:

$$
d_{\text {max }}\left(f_{k}\right) \sim 10^{-10}, \quad d_{\text {mean }}\left(f_{k}\right) \sim 10^{-15} .
$$

Results are depicted in Fig. 8 for $n_{\min }=10, n_{\max }=50, \Delta n=10$ and a convergence criterion on the residual set to $\epsilon_{\text {tol }}=10^{-4}$. The FFS algorithm needs 23 windows to cover the full frequency range, as shown in Fig. 8(a). For low frequencies, the reduced model approximation bandwidth is typically larger, the first two windows reaching up to $170 \mathrm{~Hz}$ bandwidth. At higher frequency, as the modal density increases, the bandwidth of the approximation reduces and stagnates around $40 \mathrm{~Hz}$.

The timings for the different operations performed at the 23 master and 1181 slave frequencies are detailed in Table 1. The main computational cost involves the matrix factorization performed by MUMPS in about 9 min using around $10 \mathrm{~Gb}$ of memory. The subspace computation inside the WCAWE algorithm, namely the orthogonalization process and the associated corrections, is also computationally intensive. For $n=50$, it requires around $5 \mathrm{~min}$, while the projection and solving the reduced system are minor operations in comparison. Still, the difference in timing between $n=10$ and $n=50$ pleads for the use of an adaptive approach inside each window, particularly when using large values of $n_{\max }$.

The full FFS computation with $1 \mathrm{~Hz}$ increment including pre-processing and system identification took $8 \mathrm{~h} 31 \mathrm{~min} 13 \mathrm{~s}$. Comparatively, with such a fine increment, a direct computation would have taken $\sim 179 \mathrm{~h}$. As shown in Fig. 8(b), this does not come at the price of a significant loss of accuracy. The approximate sound pressure level obtained with the FFS shows an excellent agreement with the one computed using the direct approach with a $10 \mathrm{~Hz}$ increment.

To study the influence of the subspace maximum order on the overall performance of the FFS, other computations were performed using $n_{\max }=20$ up to $n_{\max }=40$. The different timings are compared in Table 2 .

Increasing the maximum subspace order $n_{\max }$ tends to reduce the number of windows and thus the number of factorizations needed to cover the frequency range. However, the subspace computation inside the WCAWE algorithm is also computationally intensive and roughly scales with $\mathcal{O}\left(n_{\max }^{3}\right)$. The optimal value of $n_{\max }$ therefore lies in the trade-off between limiting the number of windows and ensuring that the moments computation does not hinder the full process. Speed-up factors between 14 and 21 are reported in all cases when compared to the direct approach. 

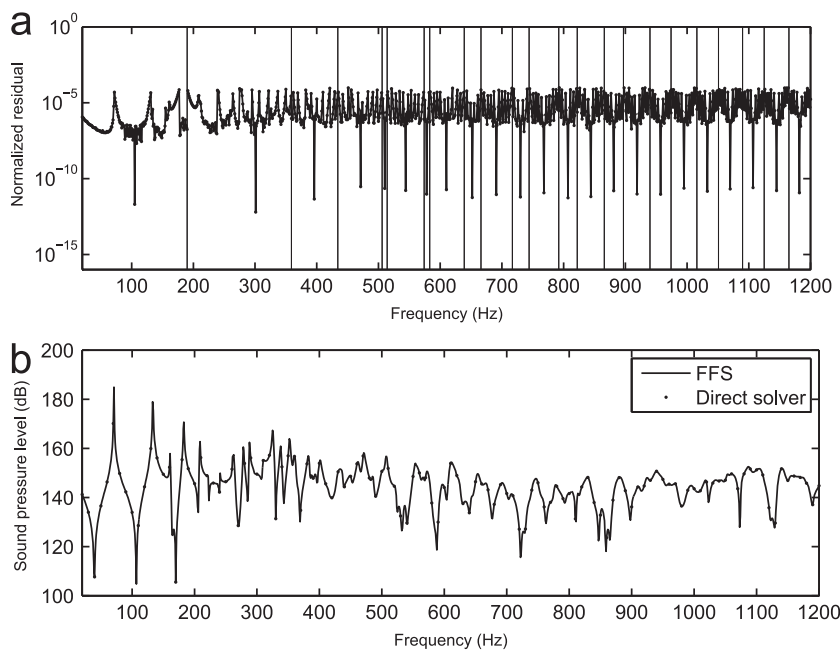

Fig. 8. Results obtained for the large scale car cavity test case with cubic admittance for $n_{\min }=10, n_{\max }=50$ and $\Delta n=10$. (a) Residual and windows (vertical lines); (b) Frequency response at the field point.

Table 1

Detailed timings (h:min:s) reported on the large scale car cavity test case for $n_{\max }=50$. The full FFS process involves 23 master frequencies and 1181 slave frequencies.

\begin{tabular}{lll}
\hline Operation & Timings & Performed at frequencies \\
\hline Taylor coefficients & $00: 00: 02$ & Master \\
Matrix factorization & $00: 09: 07$ & Master \\
Subspace computation $(n=50)$ & $00: 04: 48$ & Master \\
System assembly & $00: 00: 03$ & Slave \\
Reduced system solving $(n=10)$ & $00: 00: 02$ & Slave \\
Reduced system solving $(n=20)$ & $00: 00: 03$ & Slave \\
Reduced system solving $(n=30)$ & $00: 00: 06$ & Slave \\
Reduced system solving $(n=40)$ & $00: 00: 09$ & Slave \\
Reduced system solving $(n=50)$ & $00: 00: 12$ & Slave \\
\hline
\end{tabular}

Table 2

Timings (h:min:s) and speed-up factors reported on the large scale car cavity test case for several values of $n_{\max }$.

\begin{tabular}{lllll}
\hline$n_{\max }$ & \# of windows & Subspace timings & Total timings & Speed-up \\
\hline 20 & 61 & $00: 00: 45$ & $12: 11: 05$ & $\mathbf{1 4 . 7}$ \\
30 & 44 & $00: 01: 35$ & $10: 27: 26$ & $\mathbf{1 7 . 1}$ \\
40 & 37 & $00: 02: 53$ & $10: 46: 29$ & $\mathbf{1 6 . 6}$ \\
50 & 23 & $00: 04: 48$ & $8: 31: 13$ & $\mathbf{2 1}$ \\
\hline
\end{tabular}

\subsection{Exterior radiation problems with PML layers}

\subsubsection{Sphere with vibrating cap}

The problem geometry used for the first exterior acoustic application is depicted in Fig. 9(a). It consists of a rigid sphere with a vibrating cap. Under free-field conditions, the complex pressure at any position outside the sphere is given by

$$
p(r, \theta)=\frac{-i \rho c v_{0}(f)}{2} \sum_{n=0}^{\infty}\left[P_{n-1}(\cos \alpha)-P_{n+1}(\cos \alpha)\right] \frac{h_{n}(k r)}{h_{n}^{\prime}(k a)} P_{n}(\cos \theta),
$$

where $r$ is the distance to the evaluation point, $h_{n}$ are the spherical Hankel functions of the first kind, $P_{n}$ are the Legendre functions, $v_{0}(f)$ is the uniform normal velocity of the spherical cap and $a$ is the radius of the sphere. A complete derivation of the analytical solution in Eq. (32) can be found in [45]. To guarantee a good accuracy of the analytical solution, the infinite summation has been truncated at $2 k$, following the rule suggested by Ihlenburg [46]. 

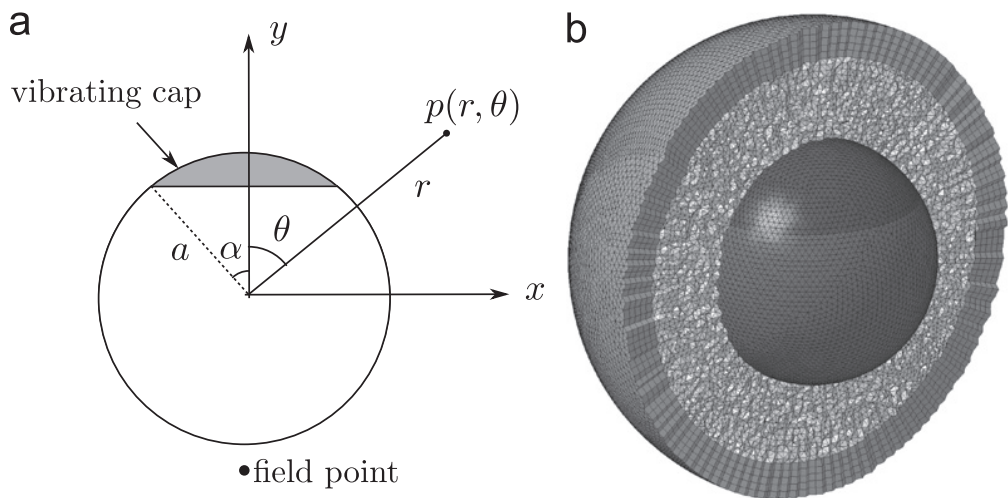

Fig. 9. Sphere with vibrating cap exterior acoustic application using locally conformal PML. (a) Sketch of the problem; (b) Mesh used for the computations (Physical tetrahedral mesh + PML pentahedral mesh).

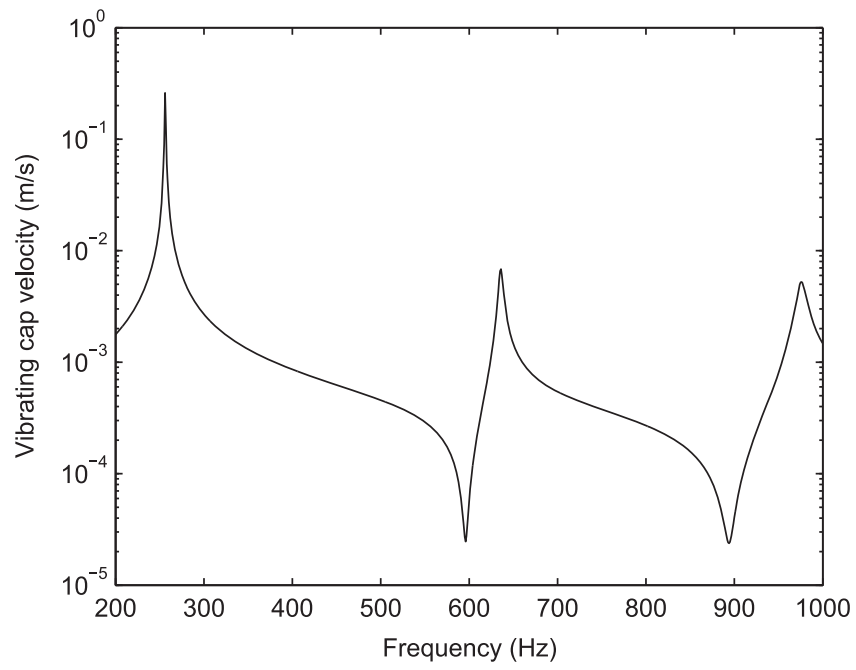

Fig. 10. Vibrating cap velocity (abs. value).

The parameters are chosen as follows. The sphere radius is $a=0.6 \mathrm{~m}$, the angle defining the vibrating cap is $\alpha=\pi / 3 \mathrm{rad}$. The sound speed and fluid density are $c=340 \mathrm{~m} / \mathrm{s}$ and $\rho=1.225 \mathrm{~kg} / \mathrm{m}^{3}$, respectively. To test the ability of the FFS to deal with right-hand sides with complex frequency dependency, the cap normal velocity is chosen as the response of a classical three degrees of freedom mass-spring-damper in series, tuned to have resonances in the frequency range of interest. The three masses are chosen respectively (in $\mathrm{kg}$ ) as $M_{1}=60, M_{2}=40$ and $M_{3}=20$, the stiffness of the three springs (in N/m) is chosen to be $K=2.7 \times 10^{5}$ and the three viscous dampers have damping constant (in N s/m) $C=20$. The three masses are subject to a unitary force. The analytical solution to this simple dynamical system can be found in [1]. The velocity of the third mass $m_{3}$ is applied as the cap normal velocity $v_{0}(f)$ (depicted in Fig. 10). The three resonances are found respectively at $f=256 \mathrm{~Hz}, f=636 \mathrm{~Hz}$ and $f=976 \mathrm{~Hz}$.

Considering the applied velocity boundary integral in the weak form (12), the right-hand side frequency dependency is rational of order 6 .

The mesh used for the computations is shown in Fig. 9(b). The physical region, comprised between the inner radiating sphere and an outer sphere of radius $1 \mathrm{~m}$, contains 233718 nodes and 1352298 tetrahedral elements. The PML elements are obtained from an automatic extrusion along the node normals of the outer sphere triangle elements, yielding 177800 pentahedral elements and 88910 additional nodes. The total number of unknowns for the resulting algebraic linear system is hence $N=322628$. The mesh is considered valid up to $1000 \mathrm{~Hz}$ using an 8 grid points per wavelength rule of thumb.

The FFS algorithm is applied in the range $[200,1000] \mathrm{Hz}$ with a $1 \mathrm{~Hz}$ frequency increment (801 individual frequencies). The corresponding non-dimensional Helmholtz number ranges between $k D=3.7$ and $k D=18.5$, where $D=1 \mathrm{~m}$. The FFS algorithm begins with the system identification procedure described in Section 4.1. The fitting procedure is applied on 
a set of 40 Chebyshev points ( 5 percent of the total number of frequencies) distributed within in the frequency interval. The outcome of the exploratory fitting phase performed on the 322628 diagonal entries is as follows:

- 66.93 percent of the entries are interpolated by a polynomial of order 2 .

- 0.02 percent of the entries are interpolated by a rational of order 3.

- 8.84 percent of the entries are interpolated by a rational of order 4.

- 18.46 percent of the entries are interpolated by a rational of order 5 .

- 5.77 percent of the entries are interpolated by a rational of order 6 .

Rational functions are required to approximate the entries arising from the PML discretization, whereas second-order polynomials represent the standard FE entries. The outcome of the final fitting phase for the 2498829 off-diagonal entries is as follows:

- 62.29 percent of the entries are interpolated by a polynomial of order 2 .

- 4.21 percent of the entries are interpolated by a rational of order 4.

- 30.62 percent of the entries are interpolated by a rational of order 5.

- 2.88 percent of the entries are interpolated by a rational of order 6 .

The fitting process is also applied on the 1602 non-zero entries of the right-hand side: all entries are interpolated with a rational order 6 . In terms of computational cost, the exploratory and final fitting phases were performed in $41 \mathrm{~s}$ and $02 \mathrm{~min}$ $46 \mathrm{~s}$, respectively. The entries associated to the physical domain, which are intrinsically of second-order frequency dependency, exhibit a maximum deviation close to machine precision. On the other hand, the frequency behavior of the PML entries is too complex to be properly captured and can only be approximated through the rational fitting procedure. This is reflected in the maximum and mean matrix entry deviations of the system entries, as defined in Eqs. (29) and (30), which are plotted as a function of frequency in Fig. 11(a). The maximum entry deviation is around $d_{\max } \sim 10^{-7}$.

Once the system is identified, the AWA algorithm is launched and the windowing process starts. The results are depicted in Fig. 11 for $n_{\min }=10, n_{\max }=20$ and $\Delta n=10$. The convergence criterion on the residual is again chosen as
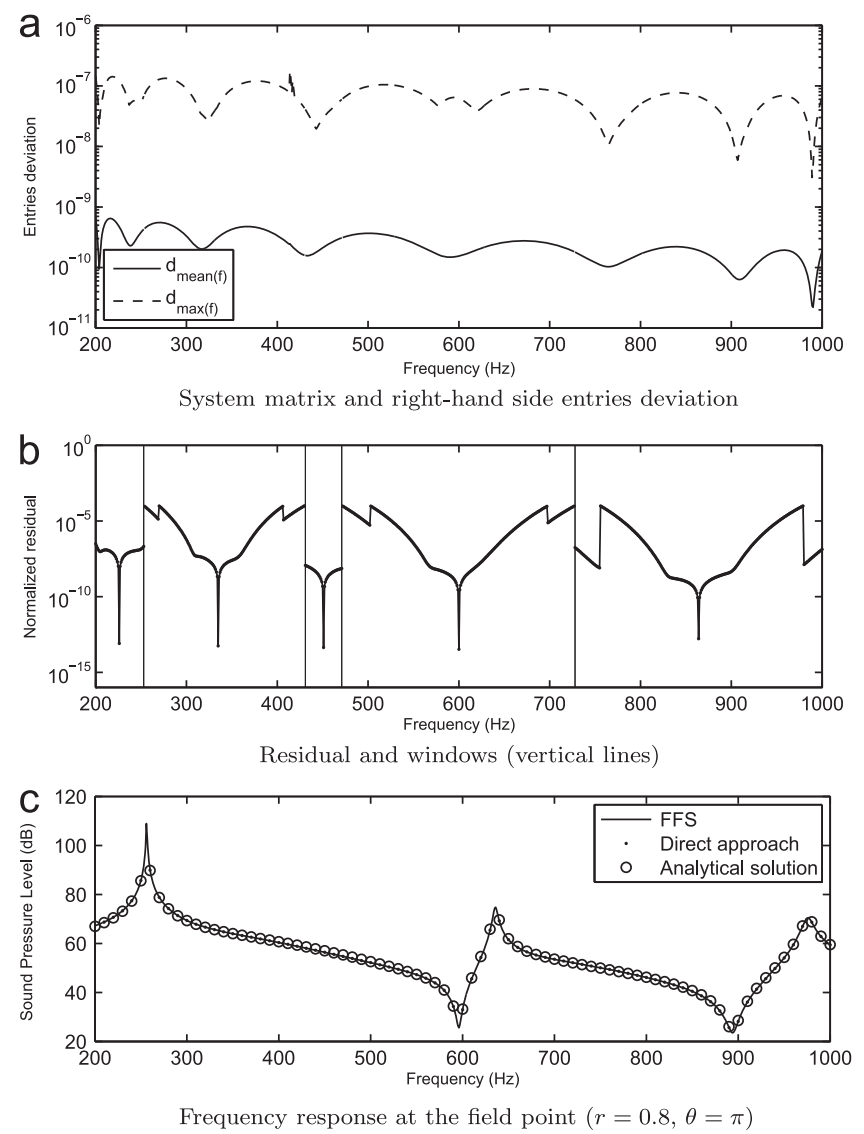

Fig. 11. Results obtained for the sphere with vibrating cap test case with PML for $n_{\min }=10, n_{\max }=20$ and $\Delta n=10$. 
$\epsilon_{\text {tol }}=10^{-4}$. Using these parameters, only 5 frequency windows are required to cover the full frequency range (see Fig. 11(b)). Each matrix factorization is performed by the MUMPS solver in 4 min $15 \mathrm{~s}$ using $6.2 \mathrm{~Gb}$ of memory. Solving the frequency sweep problem for the 801 individual frequencies using the direct approach would have taken about $57 \mathrm{~h}$. The timings of the FFS are reported in Table 2 for maximum subspace dimensions $n_{\max }=20, n_{\max }=30$ and $n_{\max }=40$. In either case, the frequency sweep problem is solved within $2 \mathrm{~h}$, yielding a speed-up factor higher than 30 (Table 3).

It should be emphasized that this test case is challenging for the non-reflecting operator, as the radiation pattern exhibits a strong directivity. The backward radiation is especially difficult to capture accurately, since it is generated by the scattering of the waves on the edges of the vibrating cap. These scattered waves typically impinge the PML at angles very far from normal incidence, for which spurious reflections are typically higher [36]. To assess the ability of the FFS to represent accurately the full dynamics of the initial problem at hand, a field point is chosen at the back of the sphere $(r=0.8, \theta=\pi)$, where the solution is the most difficult to capture.

Again, the analysis of the frequency responses demonstrates that the speed-up provided by the FFS algorithm does not come at the price of a significant loss of accuracy. Fig. 11(c) compares the sound pressure levels at the chosen field point obtained with FFS and the direct approach solved with a $10 \mathrm{~Hz}$ increment. The frequency responses compare very well along the full frequency range. The analytical solution computed with Eq. (32) is also plotted. Again, no notable differences can be observed, all sound pressure levels are in excellent agreement.

\subsubsection{Drop-shape with vibrating cap}

As a last test case, an application involving a non-spherical PML is considered. A cross-section of the computational mesh is provided in Fig. 12. The drop-shaped scatterer consists of a conic surface of height $2.2 \mathrm{~m}$ mounted on top of a unit radius half sphere centered at the origin. The physical region comprised between the scatterer and the PML contains 468402 nodes and 2690870 tetrahedral elements. The PML region obtained from a direct extrusion along the node normals of the outer physical surface contains 226030 pentahedral elements and 113025 nodes. The total number of unknowns for the resulting system matrix is hence of $N=581427$.

Except for the shape and size of the scatterer and subsequent surrounding computational domain, all parameters are similar to the previous test case. The same mesh length, fluid properties, frequency bandwidth of interest and vibrating cap velocity are applied. Also, the same parameters are considered for the system identification procedure. The fitting algorithm is applied on a set of 40 Chebyshev points distributed within the frequency interval. The statistics of the final fitting phase indicate that:

- 72.42 percent of the entries are interpolated by a polynomial of order 2 .

- 4.44 percent of the entries are interpolated by a rational of order 4 .

- 9.48 percent of the entries are interpolated by a rational of order 5 .

- 13.66 percent of the entries are interpolated by a rational of order 6 .

In comparison with the sphere test case, the fitting process is more involved. This is caused by the matrix entries associated to the non-spherical PML which seem to exhibit a more complex dependency than their spherical counterpart.

Table 3

Timings (h:min:s) and speed-up factors reported on the sphere with vibrating cap test case for several values of $n_{\max }$.

\begin{tabular}{lllll}
\hline$n_{\max }$ & \# of windows & Subspace timings & Total timings & Speed-up \\
\hline 20 & 5 & $00: 00: 30$ & $01: 30: 28$ & $\mathbf{3 7 . 8}$ \\
30 & 5 & $00: 01: 05$ & $01: 38: 14$ & $\mathbf{3 4 . 8}$ \\
40 & 5 & $00: 02: 01$ & $01: 47: 54$ & $\mathbf{3 1 . 7}$ \\
\hline
\end{tabular}

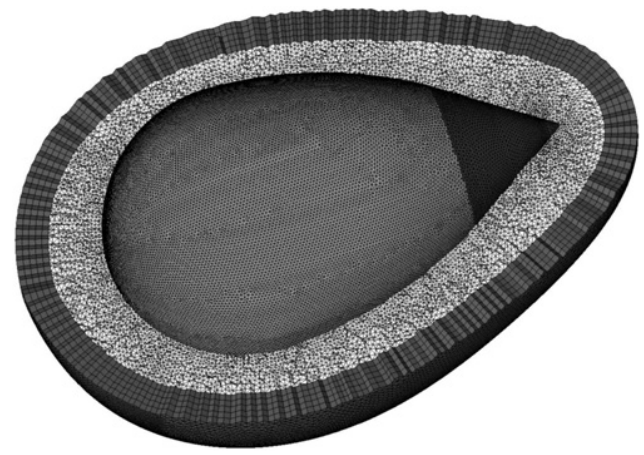

Fig. 12. Mesh cross-section of the application case consisting of the drop shape with vibrating cap using locally conformal PML: physical region (white), PML region (grey) and vibrating cap (black). 
This is in agreement with the analysis provided in Section 2.4, which establishes a clear link between the PML element distortion and the complexity of the frequency dependency of the system entries. This is reflected in the maximum and mean entries deviation of the system matrix, which are plotted as a function of frequency in Fig. 13(a). The maximum entry deviation is found to be two orders of magnitude higher than for the spherical test case $\left(d_{\max } \sim 10^{-5}\right)$. Nevertheless, in spite of this significant loss of accuracy in the system identification, the FFS results are satisfactory. The FFS timings are reported in Table 4 for maximum subspace dimensions $n_{\max }=20, n_{\max }=30$ and $n_{\max }=40$. As increasing $n_{\max }$ did not lead to a smaller number of windows ( 7 windows are needed in each case), the highest speed-up factor is obtained for the lowest subspace dimension $n_{\max }=20$. In any case, the speed-up factor is again superior to 30 . Also of interest is the comparison between the matrix factorization and the subspace computation timings. For this test case, each matrix factorization is performed by MUMPS in $9 \mathrm{~min} 33 \mathrm{~s}$. The computation of a subspace matching 20 moments at the master frequency requires only an additional 10 percent of that initial effort. This subspace can then be used to efficiently extrapolate the frequency response in the neighbourhood.

The residual history, driving the AWA bisection algorithm is plotted in Fig. 13(b) for the case $n_{\max }=20$, highlighting the frequency coverage of the 7 windows and the location of the master frequencies. In the absence of an analytical solution, the direct solution computed with a $10 \mathrm{~Hz}$ increment is used as a reference. It is compared with the FFS solution in

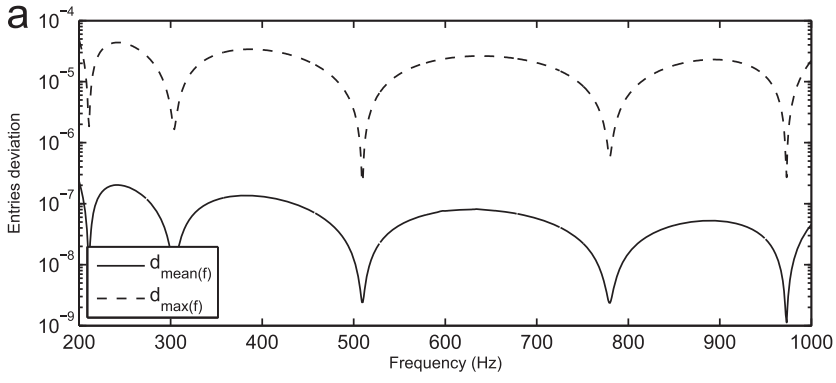

System matrix and right-hand side entries deviation
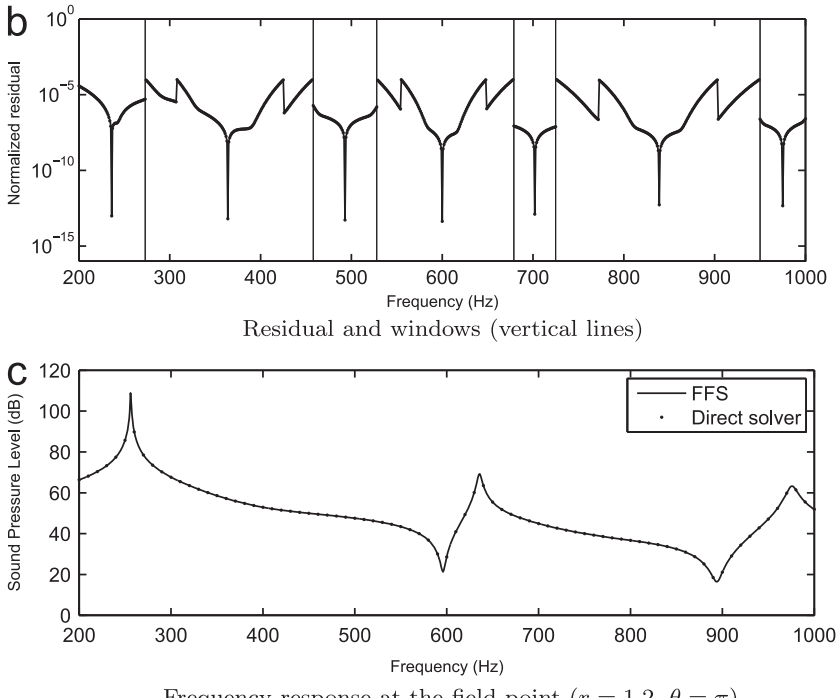

Fig. 13. Results obtained for the drop shaped scatterer with vibrating cap test case with PML for $n_{\min }=10, n_{\max }=20$ and $\Delta n=10$.

Table 4

Timings (h:min:s) and speed-up factors reported on the drop-shape with vibrating cap test case for several values of $n_{\max }$.

\begin{tabular}{lllll}
\hline$n_{\max }$ & \# of windows & Subspace timings & Total timings & Speed-up \\
\hline 20 & 7 & $00: 00: 56$ & $03: 08: 28$ & $\mathbf{3 7 . 2}$ \\
30 & 7 & $00: 01: 57$ & $03: 21: 52$ & $\mathbf{3 4 . 3}$ \\
40 & 7 & $00: 03: 37$ & $03: 40: 23$ & $\mathbf{3 1 . 4}$ \\
\hline
\end{tabular}


Fig. 13(c) for a field point located at the back of the scatterer $(r=1.2, \theta=\pi)$. The FFS frequency response is again in excellent agreement with the direct solution along the full frequency range.

\section{Conclusion}

This paper proposes an algorithm to accelerate the computation of frequency sweeps for Helmholtz finite element models which exhibit a non-second-order frequency dependency. The application of the well-conditioned asympotic waveform evaluation (WCAWE) is extended to systems with undefined frequency behavior. WCAWE, a robust algorithm which computes an orthogonal basis for the subspace of moments of a dynamical system, has been coupled with a multiexpansion points strategy to render an efficient FFS procedure. A system identification step is used to construct polynomial or rational function approximations for all system entries, which allows for the computation of the derivatives needed in the expression of the Taylor coefficients. For systems with a complicated frequency behavior, the moments calculated with the proposed approach are not exact and can only be approximated. However, the numerical examples demonstrate that substantial accelerations are possible while maintaining a high level of accuracy of the frequency response. This approach allows tackling more easily a larger set of mid-frequency applications, helping to address a broader frequency range for a given computational effort as compared to the direct solution strategy.

\section{Acknowledgments}

The authors kindly acknowledge the European Commission (EC) for the support of the FP7 Marie Curie Initial Training Network (ITN) project 214909 "Mid-Frequency", from which the first and second author hold respectively an Early Stage Researcher (ESR) and an Experienced Researcher (ER) Grant (see http://www.midfrequency.org).

\section{References}

[1] M. Mukhopadhyay, Vibrations, Dynamics and Structural Systems, Taylor \& Francis, 2000.

[2] J. Baker, P. Graves-Morris, Padé Approximants, second ed. Cambridge University Press, Cambridge, UK, 1996.

[3] P. Feldman, R.W. Freund, Efficient linear circuit analysis by Padé approximation via the Lanczos process, IEEE Transactions on Computer-Aided Design of Integrated Circuits and Systems 14 (1995) 639-649.

[4] K. Gallivan, E. Grimme, P.V. Dooren, Padé approximation of large-scale dynamic systems with Lanczos methods, IEEE Conference on Decision and Control 14 (1994) 443-448.

[5] J.I. Aliaga, D.L. Boley, R.W. Freund, V. Hernández, A Lanczos-type method for multiple starting vectors, Mathematics of Computation 69 (2000) 1577-1601.

[6] H. Liew, P.M. Pinsky, Matrix-Padé via Lanczos solutions for vibrations of fluid-structure interaction, International Journal for Numerical Methods in Engineering 84 (2010) 1183-1204.

[7] M. Kuzuoglu, R. Mittra, Finite element solution of electromagnetic problems over a wide frequency range via the Padé approximation, Computer Methods in Applied on Mechanics and Engineering 169 (1999) 263-277.

[8] Z. Bai, Y. Su, Dimension reduction of large-scale second-order dynamical systems via a second-order Arnoldi method, SIAM Journal on Scientific Computing 26 (5) (2005) 1692-1709.

[9] M.M. Wagner, P.M. Pinsky, A.A. Oberai, M. Malhotra, A Krylov subspace projection method for simultaneous solution of Helmholtz problems at multiple frequencies, Computer Methods in Applied on Mechanics and Engineering 192 (2003) 4609-4640.

[10] J.P. Tuck-Lee, P.M. Pinsky, Adaptive frequency windowing for multifrequency solutions in structural acoustics based on the matrix Padé-via-Lanczos algorithm, International Journal for Numerical Methods in Engineering 55 (2008) 728-746.

[11] R.S. Puri, D. Morrey, A.J. Bell, J.F. Durodola, E.B. Rudnyi, J.G. Korvink, Reduced order fully coupled structural-acoustic analysis via implicit moment matching, Applied Mathematical Modeling 33 (2009) 4097-4119.

[12] Y. Chahlaoui, K.A. Gallivan, A. Vandendorpe, P.V. Dooren, Model reduction of second-order systems, P. Benner, G. Golub, V. Mehrmann, D. Sorensen (Eds.), Dimension Reduction of Large-Scale Systems, Lecture Notes in Computational Science and Engineering, Vol. 45, Springer-Verlag, Berlin, Heidelberg2005, pp. 149-170.

[13] Z. Bai, K. Meerbergen, Y. Su, Arnoldi methods for structure preserving dimension reduction of second-order dynamical systems, P. Benner, G. Golub, V. Mehrmann, D. Sorensen (Eds.), Dimension Reduction of Large-Scale Systems, Lecture Notes in Computational Science and Engineering, vol. 45 Springer-Verlag, Berlin, Heidelberg2005, pp. 173-189.

[14] F. Tisseur, K. Meerbergen, A survey of the quadratic eigenvalue problem, International Journal for Numerical Methods in Engineering 55 (2000) $728-746$.

[15] K. Meerbergen, Fast frequency response computation for Rayleigh damping, International Journal for Numerical Methods in Engineering 73 (1) (2008) 96-106.

[16] Z. Bai, Y. Su, SOAR: a second-order Arnoldi method for the solution of the quadratic eigenvalue problem, SIAM Journal on Matrix Analysis and Applications 26 (3) (2005) 640-659.

[17] Z. Jia, Y. Sun, A refined second-order Arnoldi (RSOAR) method for the quadratic eigenvalue problem and implicit restarting. arXiv:1005.3947v3, 2011.

[18] Y. Su, J. Wang, X. Zeng, Z. Bai, C. Chiang, D. Zhou, SAPOR: second-order Arnoldi method for passive order reduction of RCS circuits, Proceedings of the 2004 IEEE/ACM International Conference on Computer-Aided Design, ICCAD '04, IEEE Computer Society, Washington, DC, USA, 2004, pp. 74-79.

[19] B. Salimbahrami, B. Lohmann, Order reduction of large scale second-order systems using Krylov subspace methods, Linear Algebra and its Applications 415 (2-3) (2006) 385-405.

[20] B. Lohmann, B. Salimbahrami, Reduction of Second-Order Systems Using Second-Order Krylov Subspaces, IFAC World Congress, Prague, Czech Republic, 2005.

[21] B. Salimbahrami, Structure Preserving Order Reduction of Large Scale Second Order Models, PhD Thesis, Technische Universität München, 2005.

[22] M.M. Wagner, P.M. Pinsky, M. Malhotra, Application of Padé via Lanczos approximations for efficient multifrequency solution of Helmholtz problems, Journal of the Acoustical Society of America 113 (1) (2003) 13-319.

[23] O. Ozgun, M. Kuzuoglu, Near-field performance analysis of locally-conformal perfectly matched absorbers via Monte Carlo simulations, Journal of Computational Physics 227 (2007) 1225-1245. 
[24] L.T. Pillage, R.A. Rohrer, Asymptotic waveform evaluation for timing analysis, IEEE Transactions on Computer-Aided Design 9 (4) (1990) 352-366.

[25] J.-P. Coyette, C. Lecomte, J.-L. Migeot, Calculation of vibro-acoustic frequency response functions using a single frequency boundary element solution and a Padé expansion, Acta Acoustica 85 (1999) 277-371.

[26] J. Gong, J. Volakis, AWE implementation for electromagnetic FEM analysis, Electronics Letters 32 (24) (1996) $2216-2217$.

[28] R. Slone, Fast Frequency Sweep Model Order Reduction of Polynomial Matrix Equation Resulting from Finite Element, PhD Thesis, Ohio State University, 2002.

[29] R. Slone, R. Lee, J. Lee, Well conditioned asymptotic waveform evaluation for finite elements, IEEE Transactions on Antennas and Propagation 51 (9) (2003) 2442-2447.

[30] P. Avery, C. Farhat, G. Reese, Fast frequency sweep computations using a multi-point Padé-based reconstruction method and an efficient iterative solver, International Journal for Numerical Methods in Engineering 69 (13) (2007) 2848-2875.

[31] R. Slone, R. Lee, J.-F. Lee, Multipoint Galerkin asymptotic waveform evaluation for model order reduction of frequency domain FEM electromagnetic radiation problems, IEEE Transactions on Antennas and Propagation 49 (10) (2001) 1504-1513.

[32] M.S. Lenzi, H. Beriot, M. Tournour, S. Donders, B. Pluymers, W. Desmet, On the use of model order reduction for acoustics: second-order Arnoldi and the adaptive windowing algorithm, Proceedings of the 18th International Congress on Sound E Vibration (ICSV18), Rio de Janeiro, Brazil, 2011.

[33] J.-P. Berenger, A perfectly matched layer for the absorption of electromagnetic waves, Journal of Computational Physics 114 (5) (1994) 185-200.

[34] F. Collino, P. Monk, Optimizing the perfectly matched layer, Computer Methods in Applied Mechanics and Engineering 164 (1998) 157-171.

[35] E. Turkel, A. Yefet, Absorbing PML boundary layers for wave-like equations, Applied Numerical Mathematics 27 (1998) $533-557$.

[36] A. Bermudez, L. Hervella-Nieto, A. Prieto, R. Rodriguez, An optimal perfectly matched layer with unbounded absorbing function for time-harmonic acoustic scattering problems, Journal of Computational Physics 223 (2007) 469-488.

[37] M. Lassas, E. Somersalo, Analysis of the PML equations in general convex geometry, Proceedings of the Royal Society of Edinburgh131 (2001) 1183-1207.

[38] L. Zschiedrich, R. Klose, A. Schadle, F. Schmidt, A new finite element realization of the perfectly matched layer method for Helmholtz scattering problems on polygonal domains in two dimensions, Journal of Computational and Applied Mathematics 188 (2005) $1183-1207$.

[39] O. Ozgun, M. Kuzuoglu, Non-Maxwellian locally-conformal PML absorbers for finite element truncation, IEEE Transactions on Antennas and Propagation 55 (2007) 931-937.

[40] Virtual.Lab Acoustics R10, User's Manual, LMS International, Leuven, Belgium, 2011.

[41] R.B. Platte, L.N. Trefethen, A.B.J. Kuijlaars, Impossibility of fast stable approximation of analytic functions from equispaced samples, SIAM Review 53 (2) (2011) 308-318.

[42] E.J. Grimme, Krylov Projection Methods for Model Reduction, PhD Thesis, University of Illinois at Urbana-Champaign, 1997.

[43] S. Gugercin, A. Antoulas, C. Beattie, H2-model reduction for large-scale linear systems, SIAM Journal on Matrix Analysis and Applications 30 (2) (2008) 609-638.

[44] P. Amestoy, I. Duff., L.J.Y., Multifrontal parallel distributed symmetric and unsymmetric solvers, Computer Methods in Applied Mechanical Engineering (184) (2000) 501-520.

[45] M.C. Junger, D. Feit, Sound, Structures and their Interaction, MIT Press, Cambridge, 1986.

[46] F. Ihlenburg, Finite Element Analysis of Acoustic Scattering, Springer Verlag, Berlin, 1998. 